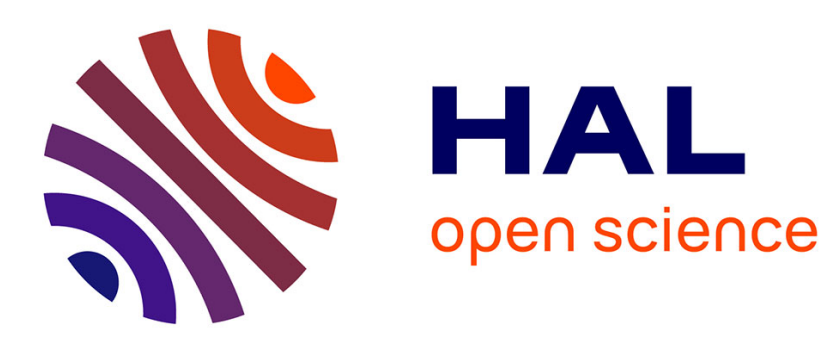

\title{
A gravity current model with capillary trapping for oil migration in multilayer geological basins
}

\author{
Clément Cancès, David Maltese
}

\section{To cite this version:}

Clément Cancès, David Maltese. A gravity current model with capillary trapping for oil migration in multilayer geological basins. SIAM Journal on Applied Mathematics, 2021, 81 (2), pp.454-484. 10.1137/19M1284233. hal-02272965v3

\section{HAL Id: hal-02272965 \\ https://hal.science/hal-02272965v3}

Submitted on 3 Jan 2021

HAL is a multi-disciplinary open access archive for the deposit and dissemination of scientific research documents, whether they are published or not. The documents may come from teaching and research institutions in France or abroad, or from public or private research centers.
L'archive ouverte pluridisciplinaire HAL, est destinée au dépôt et à la diffusion de documents scientifiques de niveau recherche, publiés ou non, émanant des établissements d'enseignement et de recherche français ou étrangers, des laboratoires publics ou privés. 


\title{
A GRAVITY CURRENT MODEL WITH CAPILLARY TRAPPING FOR OIL MIGRATION IN MULTILAYER GEOLOGICAL BASINS
}

\author{
CLÉMENT CANCĖS AND DAVID MALTESE
}

\begin{abstract}
We propose a reduced model accounting capillary trapping to simulate oil migration in geological basins made of several rock types. Our model is derived from Darcy type models thanks to Dupuit approximation and a vertical integration in each geological layer. We propose a time-implicit finite volume scheme which is shown to be unconditionally stable and to admit discrete solutions. Numerical outcomes are then provided in order to illustrate the behavior of our reduced model.
\end{abstract}

Keywords. Large scale basin modeling, multilayer Dupuit approximation, capillary trapping, Finite Volumes

AMS subjects classification. 65M12, 65M08, 76S05

\section{InTRODUCTION}

1.1. General motivations. In oil engineering, basin modelling aims at predicting the potential of a geologic area in terms of oil production. The goal is to localize and quantify the presence of hydrocarbons in the porous space of the subsoil. This knowledge can then be used to decide of the exploitation of reservoirs and to optimize the location of the wells. Reconstructing the history of oil migration is therefore an important issue.

In the geological basin context, gravity plays a major role. Hydrocarbons rise within the porous medium to the surface due to buoyancy. Along their migration, they encounter some heterogeneities - typically some changes in the rock properties - that can perturb their progression. For instance, oil can be blocked by impervious (or at least very low permeable) geological structures. But as already predicted by Berg [8] and England et al. [25], oil can also be trapped in reservoirs because of capillary barriers. Capillary barriers correspond to severe changes of the pore size as illustrated in Figure 1. At equilibrium, the capillary pressure is inversely proportional to the pore radius following Laplace's law. Therefore, heterogeneities in the pore radius distribution generate some force oriented from small pores to large pores. In the case of a discontinuous material, the resulting force is singularly located at the level of the interface between the different material, to which it is orthogonal. This resulting capillary force can equilibrate with buoyancy, and may entail a quantity of entrapped oil that remains below the capillary barrier. The effects of such capillary barriers on two-phase porous media flows was for instance studied in $[23,15]$. We refer to [8] for a more developed discussion on the microscopic phenomenons leading to capillary trapping.

In the context of basin simulation, dynamics is generally slow. Therefore, Darcean models (see for instance [7]) for immiscible two-phase flows are trustworthy. Such models yield degenerate parabolic problems set on 3-dimensional spatial domains. 

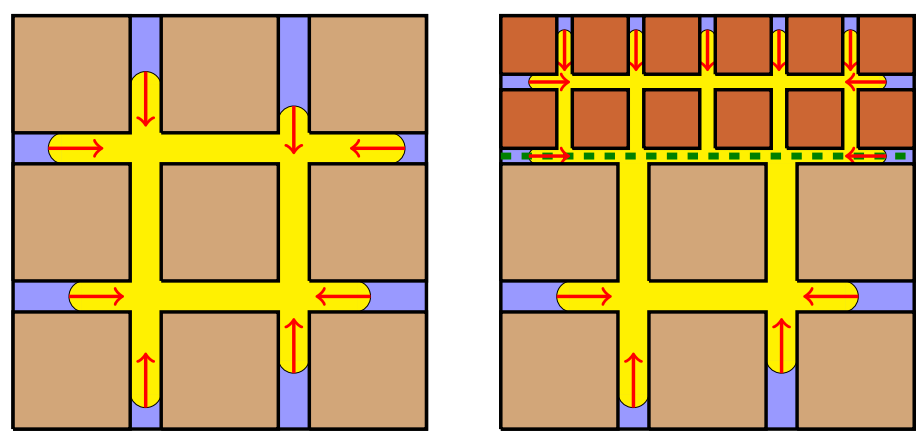

FiguRE 1. Within a homogeneous ideal porous medium with constant pore size, the resulting capillary force on a connected component of non-wetting phase vanishes (left). This is no longer true if the non-wetting phase straddles the interface between two idealized porous media with different characteristics (right): capillarity generates a force orthogonal to the interface represented by the dashed line separating the regions with pores of different sizes.

However, the large time and space scales naturally involved in basin modelling make the resolution of such models very demanding from a computational point of view. This motivates the introduction of reduced models with lower complexity.

A first class of reduced model can be obtained thanks to ray-tracing. The raytracing method constructs migration flow paths based on the top depth map of a carrier bed (see [54]). The underlying assumption is that hydrocarbon flowrates and therefore hydrocarbon saturations change very slowly as compared to the duration of simulation time-steps and that migration can therefore be modelled as a steady state process. A second method is the invasion percolation method. Percolation theory can by applied to porous media and deals with the description of interconnections of the porous and fractured network. The percolation invasion introduced in [56] is motivated by the problem of one fluid displacing another from a porous medium under the action of capillary forces. This model has then been extended in [55] by adding the effects of buoyancy. Computationally efficient algorithms have been proposed for instance in [40] for the simulation of invasion percolation.

The history of the migration is kept neither with invasion percolation nor with ray tracing since both reduced models do not keep time as a variable, the goal being to compute directly a steady state, or equivalently a (local) minimizer of the energy since immiscible two-phase flows have a gradient flow structure [17]. The energy, which is made of the gravitational potential energy and the internal energy related to capillarity, is in general non-convex in the McCann's sense [39] when the domain is heterogeneous, so that it may admit several minimizers. It is therefore necessary to compute the whole dynamics to get a trustworthy knowledge of which steady state is relevant. Moreover, reconstructing the history of the migration helps to better predict anomalous pressures in the subsoil. We refer to [48] for an extensive comparison between reduced models and full Darcy models in basin modelling.

In view of the above discussion, our goal is to get a model with reduced complexity while keeping track of the time variable. To this end, we make use of Dupuit 
approximation [24], which consists in assuming the fluid is at vertical equilibrium, so that the flow is mainly horizontal. After integrating vertically a full Darcean model, this allows to derive a reduced model where the main unknown is no longer the composition of the fluid but rather the position of the free boundaries between the different regions occupied by pure phases. This approach is classical and has been shown to be very efficient in the context of groundwater modeling for water or hydrocarbon resource management [6]. Vertically integrated models are also very popular in the community working on carbon dioxyde sequestration [47]. In [34], Huppert and Woods studied the evolution of a buoyant fluid entrapped by a impervious seal. Excess pressure originating from confinement have been considered by Nordbotten \& Celia [45] and Pegler et al. [49].

The originality of our contribution relies in leakage term (10), which differs from what exists in the literature. The leakage terms is shown to be able to reproduce both capillary trapping and excess pressures related to congestion when layers are fully saturated by one pure phase. Models with localized in space leakage modelling for instance fractures were considered by Pritchard [51], Neufeld et al. [42, 43], Kang et al. [37] or Pegler et al. [50]. Leakage across the whole surface separating the geological layers is represented by simple linear contributions in [33, 52]. Due to their simplicity, such models cannot reproduce oil-trapping, which requires the introduction of a nonlinear contribution, as for instance proposed by Woods \& Farcas [57] or Ngo et al. [44]. These two models [57, 44] are closely related to ours, with differences highlighted in the forthcoming Remark 1.1. In contributions by Hesse et al. [32], Juanes et al. [36], Zhao et al. [58], related to carbon dioxyde sequestration, capillary trapping is defined as a volume contribution that models the immobile $\mathrm{CO}_{2}$ entrapped in pores since not connected to the main (and mobile) carbon dioxyde components. Refined models obtained by relaxing the vertical equilibrium assumption are presented by Nordbotten, Celia and collaborators [46, 37], while models with diffuse interface between fluids have been proposed for instance by Gunn et al. [29], Ngo et al. [44], or Choquet et al. [20] in the context of coastal aquifers with seawater intrusion.

Our model is described in the next section, whereas its derivation is the purpose of Section 2. Before presenting the model, let us stress that it has been designed in such a way that it preserves some important stability features inherited from full Darcean models. The nonlinear stability of the model is key to guarantee its good behavior. A complete mathematical study of the model is not the purpose of this paper, where a large part is rather devoted to the construction of a finite volume scheme detailed in Section 3. The scheme relies on two-point flux approximation [26] with upstream mobilities [4, 28]. This choice is known to be suitable for such vertically integrated models, see for instance $[1,2]$ since it allows to transpose the stability of the continuous model to the discrete one. The strong stability of the numerical scheme allows to show that the corresponding nonlinear system admits solutions, which are computed thanks to a parametrization based robust Newton solver [5] allowing for large time steps. All in all, the numerical procedure appears to be very efficient to compute the long-time behavior of the model in which we are interested. Numerical results are then presented in Section 4. This study highlights in particular the fact that our model is able to capture different steady states depending on how quick is the inflow at the bottom of the computational domains. 


\subsection{Presentation of the reduced model.}

1.2.1. Geometry of the basin. As in the early work by Hunt [33], we assume that the geological basin $\Omega \subset \mathbb{R}^{d+1}(d \in\{1,2\})$ is made of the vertical superposition of $I$ vertical layers $\left(\Omega_{i}\right)_{1 \leq i \leq I}$. We assume that the interface between $\Omega_{i}$ and $\Omega_{i+1}$ can be described by $z=\bar{b}_{i}(\boldsymbol{x})$, where $z$ denotes the vertical coordinate and $\boldsymbol{x} \in \mathbb{R}^{d}$ is the horizontal coordinate. We also assume that there exist functions $b_{0}$ and $b_{I}$ corresponding to the lower and upper boundary of $\Omega_{1}$ and $\Omega_{I}$ respectively. We denote by

$$
H_{i}(\boldsymbol{x})=b_{i}(\boldsymbol{x})-b_{i-1}(\boldsymbol{x})
$$

the thickness of the $i^{\text {th }}$ layer $\Omega_{i}$. To ease the presentation, we assume that

$$
\Omega_{i}=\bigcup_{\boldsymbol{x} \in \mathcal{O}}\{\boldsymbol{x}\} \times\left(b_{i-1}(\boldsymbol{x}), b_{i}(\boldsymbol{x})\right)
$$

for some open and bounded set $\mathcal{O} \subset \mathbb{R}^{d}$.

Two incompressible and immiscible phases (oil and water) flow within each layer $\Omega_{i}$. Since oil is less dense than water, oil is located on top of the layer, while water occupies its bottom. We assume that the interface separating the oil and the water layers is sharp. The thickness of the oil layer in $\Omega_{i}$ is then denoted by $h_{i}$, and we have naturally that

$$
0 \leq h_{i}(t, \boldsymbol{x}) \leq H_{i}(\boldsymbol{x}), \quad t \geq 0, \boldsymbol{x} \in \mathcal{O} .
$$

The thickness of the water layer is then equal to $\left(H_{i}-h_{i}\right)$. The interface between the two phases is located at

$$
z=\gamma_{i}(t, \boldsymbol{x})=b_{i}(\boldsymbol{x})-h_{i}(t, \boldsymbol{x}), \quad t \geq 0, \boldsymbol{x} \in \mathcal{O} .
$$

We refer to Figure 2 for a schematic depiction of the geometry.

1.2.2. About the phase pressures. Each phase (water and oil) is assumed to have its own pressure as a consequence of their immiscibility. We assume that Dupuit's assumption is verified, i.e. vertical equilibrium holds for both phases. More precisely, water is assumed to be the wetting phase and to be hydrostatic in the whole domain $\Omega$ :

$$
p_{\mathrm{w}}(t, \boldsymbol{x}, z)=-\varrho_{\mathrm{w}} g z, \quad t \geq 0,(\boldsymbol{x}, z) \in \Omega,
$$

where $\varrho_{\alpha}$ denotes the density of the phase $\alpha$ and $g$ is the gravity constant. Oil is assumed to be hydrostatic only in the regions where it accumulates, i.e.,

$$
p_{\mathrm{o}}(t, \boldsymbol{x}, z)=-\varrho_{\mathrm{o}} g z+C_{i}(t, \boldsymbol{x}), \quad \gamma_{i}(t, \boldsymbol{x})<z<b_{i}(\boldsymbol{x}) .
$$

In the above equation, $C_{i}$ encodes the horizontal variations of the oil pressure field. Each porous medium $\Omega_{i}$ is assumed to be poorly graded, so that the capillary pressure can be assumed to be constant equal to $\pi_{i}$ as soon as both phases are present and mobile. In particular, denoting by $p_{\alpha}(t, \boldsymbol{x}, z)$ the pressure of phase $\alpha$ at time $t$ and position $(\boldsymbol{x}, z) \in \Omega$, this amounts to claim that

$$
p_{\mathrm{o}}\left(t, \boldsymbol{x}, \gamma_{i}(t, \boldsymbol{x})\right)-p_{\mathrm{w}}\left(t, \boldsymbol{x}, \gamma_{i}(t, \boldsymbol{x})\right)=\pi_{i} \quad \text { if } \quad b_{i-1}(\boldsymbol{x})<\gamma_{i}(t, \boldsymbol{x})<b_{i}(\boldsymbol{x}) .
$$

The assumption of sharpness for the interface is consistent with an assumption of negligible capillary diffusion. 


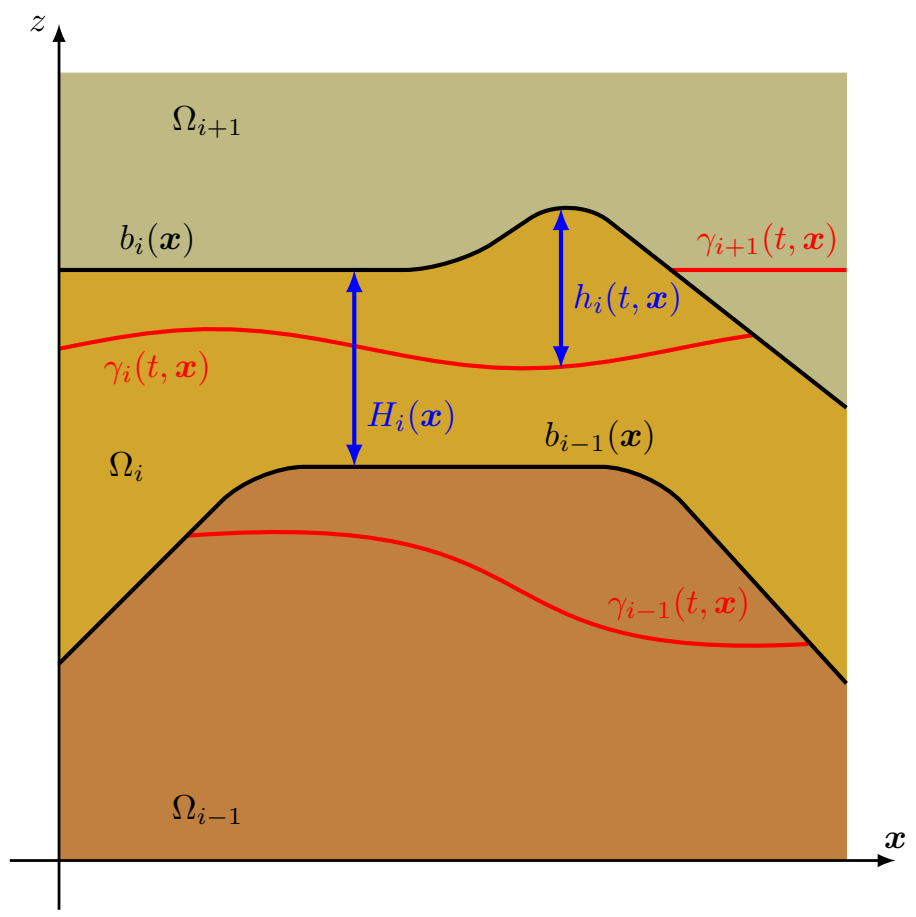

FigURE 2. Schematic representation of the multilayer geological basin. The interfaces between the $i^{\text {th }}$ and the $(i+1)^{\text {th }}$ layer is located at $z=b_{i}(\boldsymbol{x})$. Within layer $\Omega_{i}$, oil is located above the interface $z=\gamma_{i}(t, \boldsymbol{x})$, whereas water is located below.

We infer from the notion of multivalued phase pressure proposed in $[19,10]$ the conditions

$$
\begin{aligned}
p_{\mathrm{o}}\left(t, \boldsymbol{x}, b_{i}(\boldsymbol{x})\right)-p_{\mathrm{w}}\left(t, \boldsymbol{x}, b_{i}(\boldsymbol{x})\right) \leq \pi_{i} & \text { if } \quad h_{i}(t, \boldsymbol{x})=0, \\
p_{\mathrm{o}}\left(t, \boldsymbol{x}, b_{i-1}(\boldsymbol{x})\right)-p_{\mathrm{w}}\left(t, \boldsymbol{x}, b_{i-1}(\boldsymbol{x})\right) \geq \pi_{i} & \text { if } \quad h_{i}(t, \boldsymbol{x})=H_{i}(\boldsymbol{x}) .
\end{aligned}
$$

Conditions (4)-(5) can be rewritten using the formalism of maximal monotone graphs. Roughly speaking, maximal monotone graphs can be thought as an extension of the notion of monotone continuous functions allowing for vertical parts in the graph, see Figure 3 for a typical example. We refer to [12] for precise definitions. Combining (4) with (5) and using the framework of maximal monotone graphs, we obtain that

$$
p_{\mathrm{o}}\left(t, \boldsymbol{x}, \gamma_{i}(t, \boldsymbol{x})\right)-p_{\mathrm{w}}\left(t, \boldsymbol{x}, \gamma_{i}(t, \boldsymbol{x})\right) \in \tilde{\pi}_{i}\left(\boldsymbol{x}, h_{i}(t, \boldsymbol{x})\right),
$$

where, for all $\boldsymbol{x} \in \mathcal{O}, \widetilde{\pi}_{i}(\boldsymbol{x}, \cdot)$ is the maximal monotone graph defined by

$$
\tilde{\pi}_{i}(\boldsymbol{x}, h)= \begin{cases}\pi_{i} & \text { if } 0<h<H_{i}(\boldsymbol{x}), \\ \left(-\infty, \pi_{i}\right] & \text { if } h=0, \\ {\left[\pi_{i},+\infty\right)} & \text { if } h=H_{i}(\boldsymbol{x}) .\end{cases}
$$

Hence, introducing the differential of the densities $\varrho=\varrho_{\mathrm{w}}-\varrho_{\mathrm{o}}>0$, we get that

$$
p_{\mathrm{o}}(t, \boldsymbol{x}, z)-p_{\mathrm{w}}(t, \boldsymbol{x}, z)=p_{i}(t, \boldsymbol{x})+\varrho g\left(z-\gamma_{i}(t, \boldsymbol{x})\right), \quad \gamma_{i}(t, \boldsymbol{x}) \leq z \leq b_{i}(\boldsymbol{x})
$$


with

$$
p_{i}(t, \boldsymbol{x}) \in \widetilde{\pi}_{i}\left(\boldsymbol{x}, h_{i}(t, \boldsymbol{x})\right), \quad t \geq 0, \boldsymbol{x} \in \mathcal{O} .
$$

The extended capillary pressure writes $p_{i}=\pi_{i}+\widetilde{p}_{i}$, where $\widetilde{p}_{i} \geq 0$ if $h_{i}=H_{i}$ and $\widetilde{p}_{i} \leq 0$ if $h_{i}=0$ can be though as Lagrange multipliers for the constraint $h_{i} \in\left[0, H_{i}\right]$.

1.2.3. Equations governing the motion of the interfaces. We have at hand the necessary material to state the equation describing the evolution of the interfaces $\gamma_{i}$. The main unknowns are the thicknesses $\left(h_{i}\right)_{1 \leq i \leq I}$ of the oil layers. Denote by $\phi_{i}$ and $\kappa_{i}$ the porosity and the permeability of the $i^{\text {th }}$ layer (which are assumed to be constant), by $\mu_{\alpha}$ the viscosity of the phase $\alpha$, and by $\varrho=\varrho_{w}-\varrho_{o}>0$ the difference of the densities. Following the approach of Huppert and Woods [34] which relies on Dupuit's assumption on vertical equilibrium, see next section for details, we obtain the following equations for $i \in\{1, \ldots, I\}$ :

$$
\phi_{i} \partial_{t} h_{i}-\nabla \cdot\left(\frac{\kappa_{i}}{\mu_{\mathrm{o}}} h_{i} \nabla\left(p_{i}+\varrho g\left(h_{i}-b_{i}\right)\right)\right)=S_{i-1}-S_{i},
$$

where $p_{i}$ satisfies (8).

The right-hand-side in (9) represents the interaction between the different layers. More precisely, $S_{i}$ denotes the oil flux across the interface $b_{i}$ between $\Omega_{i}$ and $\Omega_{i+1}$. It is computed thanks to formula

$$
S_{i}=\frac{\kappa_{i}}{\mu_{w} \frac{\kappa_{i}}{\kappa_{i+1}} H_{i+1}+\mu_{o} h_{i}}\left(\varrho g h_{i}-\left(p_{i+1}-p_{i}\right)\right)^{+}, \quad 1 \leq i \leq I-1,
$$

where $a^{+}=\max (a, 0)$ denotes the positive part of $a \in \mathbb{R}$.

Remark 1.1. Formula (10), the derivation of which is the purpose of Section 2.3, deserves some comments.

(i) First, note that $S_{i} \geq 0$, so that oil can only rise up towards the surface. Assume that $p_{i}=\pi_{i}$ and $p_{i+1}=\pi_{i+1}$ with $\pi_{i}<\pi_{i+1}$, then $S_{i}=0$ if $h_{i} \leq$ $\frac{\pi_{i+1}-\pi_{i}}{\varrho g}$, which is the predicted thickness of the reservoir found in Berg [8]. This threshold value is classically used in ray-tracing or invasion percolation algorithms. Hence formula (10) allows to reproduce oil trapping by capillary barriers.

(ii) In [57], Woods and Farcas also propose a vertically integrated model allowing for leakage between the different layers as soon as the threshold value $\frac{\pi_{i+1}-\pi_{i}}{\varrho g}$ is overpassed. However, our formula (10) is quite different of the one proposed in [57], where the interlayer flux $S_{i}$ is not a continuous function of the entrapped oil thickness $h_{i}$. This lack of continuity may lead to the non-existence of solution to the corresponding system. In opposition, our formula (10) yields a continuous dependance of $S_{i}$ w.r.t. $h_{i}$, which is key for the analysis of the numerical scheme proposed in Section 3, see in particular the proof of Theorem 3.3.

(iii) In their paper [44], Ngo et al. also consider interlayer leakage due to capillary effects. In their approach detailed in [44, Section 4], capillary diffusion is not neglected in bulk regions, so that the oil and water phases are no longer clearly segregated. This yields a modified threshold value depending on the pore size distribution and on the permeability ratio $\kappa_{i+1} / \kappa_{i}$. The permeability ratio also plays a role in (10), but not in the threshold value $\frac{\pi_{i+1}-\pi_{i}}{\varrho g}$. 
(iv) It is worth mentioning here the contribution by Pegler et al. [50] where two porous rocks with identical properties are separated by a thin impervious but locally damaged layer. Leakage then occurs through the pointy fault. The corresponding leakage term has similarities with our terms, but differs since capillarity however plays no role. It also differs in the prefactor, see discussion below.

(v) As already mentioned, our leakage term (10) shows similarities with existing contributions. Besides the other differences pointed out previously, our leakage term involves a prefactor $\frac{\kappa_{i}}{\mu_{w} \frac{\kappa_{i}}{\kappa_{i+1}} H_{i+1}+\mu_{o} h_{i}}$ that is deduced from the one-dimensional study detailed in Section 2.3. This yields a sub-linear behavior and boundedness for the leakage term $S_{i}$ expressed as a function of $h$ in absence of congestion (i.e. when $p_{i}=\pi_{i}$ and $p_{i+1}=\pi_{i+1}$ ). In the asymptotic $h_{i} \rightarrow+\infty$, then the contributions related to capillarity vanish and the leakage term reduces to $\lim _{h_{i} \rightarrow+\infty} S_{i}=\frac{\kappa_{i}}{\mu_{o}} \varrho g$, which is exactly the vertical velocity corresponding to the gravitational transport term.

The system (8)-(10) has to be complemented with initial and boundary conditions. We assume that the initial thicknesses are given:

$$
h_{i}(0, \boldsymbol{x})=h_{i}^{0}(\boldsymbol{x}), \quad 1 \leq i \leq I, \boldsymbol{x} \in \mathcal{O} .
$$

We have to consider lateral boundary conditions for $\boldsymbol{x} \in \partial \mathcal{O}$, a boundary condition on the bottom of domain $\Omega$ for $z=b_{0}$ and a boundary condition on top of the domain $\Omega$ for $z=b_{I}$. We assume no-flux boundary conditions cross the lateral boundary, i.e.,

$$
\left(\frac{\kappa_{i}}{\mu_{\mathrm{o}}} h_{i} \nabla\left(p_{i}+\varrho g\left(h_{i}-b_{i}\right)\right)\right) \cdot \boldsymbol{n}_{\mathcal{O}}=0 \quad \text { on } \partial \mathcal{O},
$$

where $\boldsymbol{n}_{\mathcal{O}}$ denotes the normal to $\partial \mathcal{O}$ outward w.r.t. $\mathcal{O}$. Imposing top and bottom boundary conditions are needed to prescribe the extremal fluxes $S_{0}$ and $S_{I}$. In order to simplify the stability analysis of the Finite Volume scheme carried out in Section 3.3, we will assume that the system is isolated, i.e.,

$$
S_{0}(t, \boldsymbol{x})=S_{I}(t, \boldsymbol{x})=0, \quad t \geq 0, \boldsymbol{x} \in \mathcal{O} .
$$

but other boundary conditions will be used in the numerical simulations.

1.2.4. Energy stability of the model. The goal of this section is to show that our reduced model preserves an important feature of Darcean immiscible incompressible two-phase flows, namely the decay of the energy along time [17, 18, 41].

Denote by $\boldsymbol{h}=\left(h_{i}\right)_{1 \leq i \leq I}$ and $\boldsymbol{p}=\left(p_{i}\right)_{1 \leq i \leq I}$. The energy functional corresponding to the reduced model (8) $-(13)$ writes

$$
\mathfrak{E}(\boldsymbol{h})=\sum_{i=1}^{I} \int_{\mathcal{O}}\left(\pi_{i} h_{i}+\frac{1}{2} \varrho g\left(b_{i}-h_{i}\right)^{2}\right) \phi_{i} \mathrm{~d} \boldsymbol{x} .
$$

Proposition 1.2. Let $(\boldsymbol{h}, \boldsymbol{p})$ be a smooth solution to (8)-(13) then

$$
\frac{\mathrm{d}}{\mathrm{d} t} \mathfrak{E}(\boldsymbol{h}) \leq 0 .
$$

Proof. Multiplying equation (9) by $p_{i}+\varrho g\left(h_{i}-b_{i}\right)$, integrating over $\mathcal{O}$ and summing over $i$ yield

$$
A+B+C=0
$$


where, using the boundary conditions (12) and (13), we have set

$$
\begin{aligned}
A & =\sum_{i=1}^{I} \int_{\mathcal{O}} \partial_{t} h_{i}\left(p_{i}+\varrho g\left(h_{i}-b_{i}\right)\right) \phi_{i} \mathrm{~d} \boldsymbol{x} \\
B & =\sum_{i=1}^{I} \int_{\mathcal{O}} \frac{\kappa_{i}}{\mu_{\mathrm{o}}} h_{i}\left|\nabla\left(p_{i}+\varrho g\left(h_{i}-b_{i}\right)\right)\right|^{2} \mathrm{~d} \boldsymbol{x}, \\
C & =\sum_{i=1}^{I-1} \int_{\mathcal{O}} S_{i}\left(p_{i}-p_{i+1}+\varrho g\left(h_{i}-h_{i+1}-\left(b_{i}-b_{i+1}\right)\right)\right) \mathrm{d} \boldsymbol{x} .
\end{aligned}
$$

In view of relation (8), either $\partial_{t} h_{i}=0$ or $p_{i}=\pi_{i}$, hence $A=\frac{\mathrm{d}}{\mathrm{d} t} \mathfrak{E}(\boldsymbol{h})$. Since $B \geq 0$, it only remains to check that $C \geq 0$ to conclude the proof. It follows from the definition (10) of $S_{i}$ that

$$
\begin{aligned}
C=\sum_{i=1}^{I-1} \int_{\mathcal{O}} \frac{\kappa_{i}}{\mu_{w} \frac{\kappa_{i+1}}{\kappa_{i}} H_{i+1}+\mu_{o} h_{i}}\left(\left(\varrho g h_{i}-\left(p_{i+1}-\right.\right.\right. & \left.\left.\left.p_{i}\right)\right)^{+}\right)^{2} \mathrm{~d} \boldsymbol{x} \\
& +\sum_{i=1}^{I-1} \int_{\mathcal{O}} S_{i}\left(b_{i+1}-b_{i}-h_{i+1}\right) .
\end{aligned}
$$

The first term in the right-hand side is obviously non-negative, as well as the second term since $h_{i+1} \leq H_{i+1}=b_{i+1}-b_{i}$ and $S_{i} \geq 0$.

Proposition 1.2 shows that $\mathfrak{E}(\boldsymbol{h})$ is a Lyapunov functional for our model when the system is isolated: no-flux boundary conditions across the lateral boundaries and $S_{0}=S_{I}=0$. We will pay attention in Section 3 to propose a Finite Volume scheme that preserves this property at the discrete level.

\section{Derivation of the Reduced MOdel}

The goal of this section is the formal justification of model (8)-(10). This will be done starting from a well-established Darcean model for immiscible incompressible two-phase flows.

2.1. Starting from a full Darcean model. Our starting point is a classical incompressible immiscible two-phase flow problem

$$
\phi_{i} \partial_{t} s_{\mathrm{o}}+\nabla_{(\boldsymbol{x}, z)} \cdot \boldsymbol{v}_{\mathrm{o}}=0 \quad \text { in } \mathbb{R}_{+} \times \Omega_{i}, i \in\{1, \ldots, I\},
$$

where the filtration velocity $\boldsymbol{v}_{\mathrm{O}}$ is related to the oil pressure through the DarcyMuskat law

$$
\boldsymbol{v}_{\alpha}=-\frac{k_{\alpha, i}\left(s_{\alpha}\right)}{\mu_{\alpha}} \kappa_{i} \nabla_{(\boldsymbol{x}, z)}\left(p_{\alpha}+\varrho_{\alpha} g z\right), \quad \alpha \in\{\mathrm{o}, \mathrm{w}\}
$$

In the two above formulas (15)-(16), we denoted the $(d+1)$ dimensional differential divergence and gradient by $\nabla_{(\boldsymbol{x}, z)} \cdot$ and $\nabla_{(\boldsymbol{x}, z)}$ in order to stress the difference with the $d$-dimensional operators $\nabla$ and $\nabla$. used in the reduced model (9). The water pressure is assumed to be hydrostatic in the whole $\Omega$, i.e., (2) holds. The relative permeability functions $k_{\mathrm{o}, i}$ are nondecreasing and satisfy $k_{\mathrm{o}, i}(0)=0, k_{\mathrm{o}, i}(1)=1$. Finally, the problem is closed thanks to a capillary pressure relation with capillary pressures depending essentially not on the saturation but only on the layer $i$ : the porous media are assumed to be infinitely poorly graded [7]. Following the previous 
works of $[53,13,16,19]$, the capillary pressure function is extended into a maximal monotone graph, leading to

$$
p_{\mathrm{o}}-p_{\mathrm{w}} \in \check{\pi}_{i}\left(s_{\mathrm{o}}\right) \quad \text { in } \mathbb{R}_{+} \times \Omega_{i}
$$

where

$$
\check{\pi}_{i}(s)= \begin{cases}\left(-\infty, \pi_{i}\right] & \text { if } s=0 \\ \pi_{i} & \text { if } 0<s<1 \\ {\left[\pi_{i},+\infty\right)} & \text { if } s=1\end{cases}
$$

for some constant $\pi_{i} \in \mathbb{R}$.

Using (2) and (17), Eq. (15) reduces to the Hele-Shaw type problem

$$
\phi_{i} \partial_{t} s_{\mathrm{o}}-\nabla_{(\boldsymbol{x}, z)} \cdot\left(\frac{k_{\mathrm{o}, i}\left(s_{\mathrm{o}}\right)}{\mu_{\mathrm{o}}} \kappa_{i} \nabla_{(\boldsymbol{x}, z)}\left(\zeta+\left(\varrho_{\mathrm{o}}-\varrho_{\mathrm{w}}\right) g z\right)\right)=0 \quad \text { in } \mathbb{R}_{+} \times \Omega_{i},
$$

with $\zeta(t, \boldsymbol{x}, z) \in \check{\pi}_{i}\left(s_{\mathrm{o}}(t, \boldsymbol{x}, z)\right)$. The diffusion is degenerated so that one can reasonably assume that both phases are disjointed, i.e., $s_{\alpha} \in\{0,1\}$ a.e. in $\mathbb{R}_{+} \times \Omega$. Since $\varrho_{\mathrm{o}}<\varrho_{\mathrm{w}}$, it is expected that $s_{\mathrm{O}}=1$ on top of the layers $\Omega_{i}$, while $s_{\mathrm{W}}=1$ in the bottom of the layers. We suppose that the two phases are separated by a sharp interface located at $z=\gamma_{i}(t, \boldsymbol{x}) \in\left[b_{i-1}(\boldsymbol{x}), b_{i}(\boldsymbol{x})\right]$. Denoting by

$$
\Omega_{\mathrm{o}, i}^{t}=\bigcup_{\boldsymbol{x} \in \mathcal{O}}\{\boldsymbol{x}\} \times\left(\gamma_{i}(t, \boldsymbol{x}), b_{i}(\boldsymbol{x})\right), \quad \text { and } \quad Q_{\mathrm{o}}=\bigcup_{t \in(0, T)}\left(\{t\} \times \bigcup_{1 \leq i \leq I} \Omega_{\mathrm{o}, i}^{t}\right),
$$

we assume that for all $t \geq 0$,

$$
s_{\mathrm{o}}(t, \boldsymbol{x}, z)= \begin{cases}1 & \text { if }(\boldsymbol{x}, z) \in \bigcup_{1 \leq i \leq I} \Omega_{\mathrm{o}, i}^{t}, \\ 0 & \text { otherwise. }\end{cases}
$$

and that the oil pressure is hydrostatic in $Q_{\mathrm{o}}$, i.e.,

$$
p_{\mathrm{o}}(t, \boldsymbol{x}, z)=-\varrho_{\mathrm{o}} g z+C_{i}(t, \boldsymbol{x}), \quad \forall t \in \mathbb{R}_{+}, \forall(\boldsymbol{x}, z) \in \Omega_{\mathrm{o}, i}^{t} .
$$

The ansatz (19)-(20) together with hydrostatic water pressure (2) and mainly horizontal interfaces $b_{i}$ are at the basis of the derivation of the vertically integrated equations to be described in the next section.

2.2. Derivation of the vertically integrated equations. We focus now on the derivation of vertically integrated equations for the evolution of the oil thicknesses $h_{i}$. For the sake of simplicity, we do not detail the formal asymptotics that lead to our model (as for instance in [35]), and we rather just base our presentation on the ansatz (19)-(20). The calculations presented in this section are mainly standard. They are detailed here for the sake of completeness.

We are interested in the evolution of the interfaces $\gamma_{i}(t, \boldsymbol{x})$. The ansatz about the strong separation of the oil and water phases in each layer detailed above yield

$$
h_{i}(t, \boldsymbol{x})=b_{i}(\boldsymbol{x})-\gamma_{i}(t, \boldsymbol{x})=\int_{b_{i-1}(\boldsymbol{x})}^{b_{i}(\boldsymbol{x})} s_{\mathrm{O}}(t, \boldsymbol{x}, z) \mathrm{d} z, \quad \forall(t, \boldsymbol{x}) \in \mathbb{R}_{+} \times \mathcal{O} .
$$

Therefore, using (15), we obtain that

$$
\phi_{i} \partial_{t} \gamma_{i}=-\phi_{i} \partial_{t} h_{i}=-\int_{b_{i-1}}^{b_{i}} \phi_{i} \partial_{t} s_{\mathrm{o}}(t, \boldsymbol{x}, z) \mathrm{d} z=\int_{b_{i-1}}^{b_{i}} \nabla_{(\boldsymbol{x}, z)} \cdot \boldsymbol{v}_{\mathrm{o}} \mathrm{d} z
$$


Since $s_{\mathrm{o}}(t, \boldsymbol{x}, z)=0$ if $z \in\left(b_{i-1}(\boldsymbol{x}), \gamma_{i}(t, \boldsymbol{x})\right)$ and since $k_{\mathrm{o}, i}(0)=0$, it follows from (16) that $\boldsymbol{v}_{\mathrm{o}}(t, \boldsymbol{x}, z)=\mathbf{0}$, then it is in particular divergence free. Therefore, one can rewrite

$$
-\phi_{i} \partial_{t} h_{i}=\int_{\gamma_{i}(t, \boldsymbol{x})}^{b_{i}(\boldsymbol{x})} \nabla_{(\boldsymbol{x}, z)} \cdot \boldsymbol{v}_{\mathrm{o}}(t, \boldsymbol{x}, z) \mathrm{d} z .
$$

Splitting the oil velocity $\boldsymbol{v}_{\mathrm{o}}$ into its horizontal part $\boldsymbol{v}_{\mathrm{o}}^{\boldsymbol{x}}$ and its vertical part $v_{\mathrm{o}}^{z} \boldsymbol{e}_{z}$, we get that

$$
\phi_{i} \partial_{t} \gamma_{i}(t, \boldsymbol{x})=\int_{\gamma_{i}(t, \boldsymbol{x})}^{b_{i}(\boldsymbol{x})} \nabla \cdot \boldsymbol{v}_{\mathrm{o}}^{\boldsymbol{x}}(t, \boldsymbol{x}, z) \mathrm{d} z+v_{\mathrm{o}}^{z}\left(t, \boldsymbol{x}, b_{i}(\boldsymbol{x})\right)-v_{\mathrm{o}}^{z}\left(t, \boldsymbol{x}, \gamma_{i}(t, \boldsymbol{x})\right) .
$$

In the case where $b_{i}$ varies slowly (i.e., if the interface is mainly horizontal, which is classically assumed in the Dupuit-Forchheimer regime, see for instance [6]), then the quantity $v_{\mathrm{o}}^{z}\left(t, \boldsymbol{x}, b_{i}(\boldsymbol{x})\right)$ can be seen as a the flux of oil leaking from $\Omega_{i}$ to $\Omega_{i+1}$ that we denote by $S_{i}(t, \boldsymbol{x})$ in what follows. The obtention of a closure relation between for $S_{i}$ is the purpose of the forthcoming Section 2.3. If the interface $\gamma_{i}$ separating the phases is mainly flat, the hydrostatic pressure profiles (2) and (20) fit with the Dupuit Forchheimer assumption, which claims that the flow is mainly horizontal. Our formal derivation does not require the assumption that $\gamma_{i}$ has small spatial variations, but this assumption is needed if one derives the model thanks to an asymptotic expansion similar to the one proposed in [35].

It appears that

$$
\boldsymbol{v}_{\mathrm{o}}^{\boldsymbol{x}}(t, \boldsymbol{x}, z)=-\frac{\kappa_{i}}{\mu_{\mathrm{o}}} \nabla p_{\mathrm{o}}(t, \boldsymbol{x}, z)
$$

is constant w.r.t. $z \in\left(b_{i-1}(\boldsymbol{x}), \gamma_{i}(t, \boldsymbol{x})\right)$ where $s_{\mathrm{O}}=1$ - thus so does $\nabla \cdot \boldsymbol{v}_{\mathrm{o}}^{\boldsymbol{x}}(t, \boldsymbol{x})$ since, thanks to (16) and (20),

$$
\partial_{z} \boldsymbol{v}_{\mathrm{o}}^{\boldsymbol{x}}(t, \boldsymbol{x}, z)=-\frac{\kappa_{i}}{\mu_{\mathrm{o}}} \nabla \partial_{z} p_{\mathrm{o}}(t, \boldsymbol{x}, z)=\frac{\kappa_{i}}{\mu_{\mathrm{o}}} \nabla \varrho_{o} g=0 .
$$

Therefore, we obtain

$$
\begin{aligned}
\int_{\gamma_{i}(t, \boldsymbol{x})}^{b_{i}(\boldsymbol{x})} \nabla \cdot \boldsymbol{v}_{\mathrm{o}}^{\boldsymbol{x}}(t, \boldsymbol{x}) \mathrm{d} z & =\nabla \cdot \int_{\gamma_{i}(t, \boldsymbol{x})}^{b_{i}(\boldsymbol{x})} \boldsymbol{v}_{\mathrm{o}}^{\boldsymbol{x}}(t, \boldsymbol{x}) \mathrm{d} z+\boldsymbol{v}_{\mathrm{o}}^{\boldsymbol{x}}(t, \boldsymbol{x}) \cdot \nabla\left(\gamma_{i}(t, \boldsymbol{x})-b_{i}(\boldsymbol{x})\right) \\
& =-\nabla \cdot\left(\frac{\kappa_{i}}{\mu_{\mathrm{o}}} h_{i}(t, \boldsymbol{x}) \nabla p_{\mathrm{o}}(t, \boldsymbol{x})\right)+\boldsymbol{v}_{\mathrm{o}}^{\boldsymbol{x}}(t, \boldsymbol{x}) \cdot \nabla\left(\gamma_{i}(t, \boldsymbol{x})-b_{i}(\boldsymbol{x})\right) .
\end{aligned}
$$

The vectors $\boldsymbol{n}_{b_{i}}=\left(\begin{array}{c}-\nabla b_{i} \\ 1\end{array}\right)$ and $\boldsymbol{n}_{\gamma_{i}}=\left(\begin{array}{c}-\nabla \gamma_{i} \\ 1\end{array}\right)$ are orthogonal to the hypersurfaces $\left\{\left(\boldsymbol{x}, b_{i}(\boldsymbol{x})\right), \boldsymbol{x} \in \mathcal{O}\right\}$ and $\left\{\left(\boldsymbol{x}, \gamma_{i}(t, \boldsymbol{x})\right), \boldsymbol{x} \in \mathcal{O}\right\}$ respectively. Equation (21) rewrites

$$
\phi_{i} \partial_{t} h_{i}-\nabla \cdot\left(\frac{\kappa_{i}}{\mu_{\mathrm{o}}} h_{i} \nabla p_{o}\right)=\boldsymbol{v}_{\mathrm{o}} \cdot\left(\boldsymbol{n}_{\gamma_{i}}-\boldsymbol{n}_{b_{i}}\right) .
$$

As a consequence of (17)-(18), the capillary pressure $p_{o}-p_{w}$ in $\Omega_{i}$ is greater than $\pi_{i}$ if $s_{\mathrm{O}}=1$ and smaller than $\pi_{i}$ if $s_{\mathrm{o}}=0$. Then capillary pressure is equal to $\pi_{i}$ at the interface between the two phases provided this interface is inside $\Omega_{i}$ :

$$
p_{\mathrm{o}}\left(t, \boldsymbol{x}, \gamma_{i}(t, \boldsymbol{x})\right)-p_{\mathrm{w}}\left(t, \boldsymbol{x}, \gamma_{i}(t, \boldsymbol{x})\right)=\pi_{i}, \quad \forall(t, \boldsymbol{x}) \text { s.t. } b_{i-1}(\boldsymbol{x})<\gamma_{i}(t, \boldsymbol{x})<b_{i}(\boldsymbol{x}) .
$$

In the case where $\gamma_{i}(t, \boldsymbol{x})=b_{i}(\boldsymbol{x})$, meaning that there is no oil in the column $\{\boldsymbol{x}\} \times\left(b_{i-1}(\boldsymbol{x}), b_{i}(\boldsymbol{x})\right)$ at time $t$, then (17)-(18) leads to

$$
p_{\mathrm{o}}\left(t, \boldsymbol{x}, \gamma_{i}(t, \boldsymbol{x})\right)-p_{\mathrm{w}}\left(t, \boldsymbol{x}, \gamma_{i}(t, \boldsymbol{x})\right) \leq \pi_{i} \quad \forall(t, \boldsymbol{x}) \text { s.t. } h_{i}(t, \boldsymbol{x})=0 .
$$


Let $\boldsymbol{x} \in \mathcal{O}$, then assume that the $\gamma_{i}(\boldsymbol{x}) \in\left(b_{i-1}(\boldsymbol{x}), b_{i}(\boldsymbol{x})\right)$. Similarly, if $\gamma_{i}(t, \boldsymbol{x})=$ $b_{i-1}(\boldsymbol{x})$, one obtains

$$
p_{\mathrm{o}}\left(t, \boldsymbol{x}, \gamma_{i}(t, \boldsymbol{x})\right)-p_{\mathrm{w}}\left(t, \boldsymbol{x}, \gamma_{i}(t, \boldsymbol{x})\right) \geq \pi_{i}, \quad \forall(t, \boldsymbol{x}) \text { s.t. } h_{i}(t, \boldsymbol{x})=H_{i}(\boldsymbol{x}) .
$$

The relations $(23)-(25)$ can be summarized in the compact form

$$
p_{\mathrm{o}}\left(t, \boldsymbol{x}, \gamma_{i}(t, \boldsymbol{x})\right)-p_{\mathrm{w}}\left(t, \boldsymbol{x}, \gamma_{i}(t, \boldsymbol{x})\right) \in \tilde{\pi}_{i}\left(\boldsymbol{x}, h_{i}(t, \boldsymbol{x})\right),
$$

where $\tilde{\pi}_{i}(\boldsymbol{x}, \cdot)$ is the maximal monotone graph defined by $(7)$.

Let $t \geq 0, \boldsymbol{x} \in \mathcal{O}$ and let $z \in\left(\gamma_{i}(t, \boldsymbol{x}), b_{i}(\boldsymbol{x})\right)$, then thanks to (20), one has

$$
\begin{aligned}
p_{\mathrm{O}}(t, \boldsymbol{x}, z) & =p_{\mathrm{o}}\left(t, \boldsymbol{x}, \gamma_{i}(t, \boldsymbol{x})\right)-\left(z-\gamma_{i}(t, \boldsymbol{x})\right) \varrho_{\mathrm{o}} g \\
& =p_{\mathrm{w}}\left(t, \boldsymbol{x}, \gamma_{i}(t, \boldsymbol{x})\right)+p_{i}(t, \boldsymbol{x})-\left(z-\gamma_{i}(t, \boldsymbol{x})\right) \varrho_{\mathrm{o}} g
\end{aligned}
$$

for some $p_{i}(t, \boldsymbol{x}) \in \widetilde{\pi}_{i}\left(\boldsymbol{x}, h_{i}(t, \boldsymbol{x})\right)$. Using the expression (2) of the water pressure, one gets

$$
p_{\mathrm{o}}(t, \boldsymbol{x}, z)=p_{i}(t, \boldsymbol{x})+\left(\rho_{\mathrm{w}}-\rho_{\mathrm{o}}\right) g\left(h_{i}(t, \boldsymbol{x})-b_{i}(\boldsymbol{x})\right)-\rho_{\mathrm{o}} g z
$$

for all $z \in\left(\gamma_{i}(t, \boldsymbol{x}), b_{i}(\boldsymbol{x})\right)$. In particular, we obtain the

$$
\nabla p_{\mathrm{o}}=\nabla\left(p_{i}+\varrho g\left(h_{i}-b_{i}\right)\right) \quad \text { on } \Omega_{\mathrm{o}, i}^{t}
$$

Using (26) in (22) yields

$$
\phi_{i} \partial_{t} h_{i}-\nabla \cdot\left(\frac{\kappa_{i}}{\mu_{\mathrm{o}}} h_{i} \nabla\left(p_{i}+\varrho g\left(h_{i}-b_{i}\right)\right)\right)=\boldsymbol{v}_{\mathrm{o}} \cdot\left(\boldsymbol{n}_{\gamma_{i}}-\boldsymbol{n}_{b_{i}}\right) .
$$

We have recovered the left-hand side of (9).

Concerning the right-hand side appearing in (27), the term $S_{i}:=\boldsymbol{v}_{\mathrm{o}} \cdot \boldsymbol{n}_{b_{i}}$ shall be interpreted as the oil flux between $\Omega_{i}$ and $\Omega_{i+1}$ leaving $\Omega_{\mathrm{o}, i}^{t}$ from above, whereas $\boldsymbol{v}_{\mathrm{o}} \cdot \boldsymbol{n}_{\gamma_{i}}$ correspond to the oil flux entering $\Omega_{\mathrm{o}, i}^{t}$ from below. For simplicity, we assume that

$$
\boldsymbol{v}_{\mathrm{o}}(t, \boldsymbol{x}) \cdot \boldsymbol{n}_{\gamma_{i}}(t, \boldsymbol{x})=S_{i-1}(t, \boldsymbol{x}),
$$

neglecting the delay corresponding to the time oil needs to reach $\gamma_{i}$ from $b_{i-1}$. This completes the derivation of (9). Our next section is devoted to the derivation of the closure relation (10).

2.3. Flux exchange term $S_{i}$ between two layers. The formula (10) for the interlayer fluxes relies on a purely one-dimensional study in the vertical direction.

We first need to relax the assumption on hydrostatic phase pressures (2) and (20) since it implies through (16) that the vertical component of $\boldsymbol{v}_{\mathrm{O}}$ is zero. We still assume that $z \mapsto p_{\mathrm{o}}(t, \boldsymbol{x}, z)$ and $z \mapsto p_{\mathrm{w}}(t, \boldsymbol{x}, z)$ are piecewise affine, but rather than looking for hydrostatic profiles, we look for profiles for which the capillary pressure remains constant equal to $p_{i} \in \tilde{\pi}_{i}\left(\boldsymbol{x}, h_{i}(t, \boldsymbol{x})\right)$ for $z \in\left(\gamma_{i}(t, \boldsymbol{x}), b_{i}(\boldsymbol{x})\right)$, i.e.,

$$
\left\{\begin{array}{l}
p_{\mathrm{w}}(t, \boldsymbol{x}, z)=\left(\alpha_{i}(t, \boldsymbol{x})-\varrho_{\mathrm{o}} g\right)\left(z-b_{i}(\boldsymbol{x})\right)+\beta_{i}(t, \boldsymbol{x}), \\
p_{\mathrm{o}}(t, \boldsymbol{x}, z)=p_{i}+\left(\alpha_{i}(t, \boldsymbol{x})-\varrho_{\mathrm{o}} g\right)\left(z-b_{i}(\boldsymbol{x})\right)+\beta_{i}(t, \boldsymbol{x}) .
\end{array}\right.
$$

Above the interface $b_{i}$, we also assume a linear profile of both phase pressures:

$$
\left\{\begin{array}{l}
p_{\mathrm{w}}(z)=\left(\alpha_{i+1}-\varrho_{\mathrm{w}} g\right)\left(z-b_{i}\right)+\beta_{i+1}, \\
p_{\mathrm{o}}(z)=p_{i+1}+\left(\alpha_{i+1}-\varrho_{\mathrm{w}} g\right)\left(z-b_{i}\right)+\beta_{i+1},
\end{array} \quad z \in\left(b_{i}, b_{i+1}\right),\right.
$$


where $p_{i+1}(t, \boldsymbol{x}) \in \tilde{\pi}_{i+1}\left(\boldsymbol{x}, h_{i+1}(t, \boldsymbol{x})\right)$. The continuity of the oil pressure at $z=$ $b_{i}(\boldsymbol{x})$ writes

$$
p_{i}+\beta_{i}=p_{i+1}+\beta_{i+1} .
$$

The total flux $\boldsymbol{v}_{\mathrm{O}}+\boldsymbol{v}_{\mathrm{w}}$ is divergence free. We assume that its vertical contribution is equilibrated, i.e., $v_{\mathrm{o}}^{z}+v_{\mathrm{w}}^{z}$ does not depend on $z$. As a consequence of the DarcyMuskat law (16), and since $s_{\mathrm{O}} \approx 1$ (resp. $s_{\mathrm{O}} \approx 0$ ) below (resp. above) the interface $b_{i}$, the total flux $v_{\mathrm{o}}^{z}+v_{\mathrm{w}}^{z}$ is mainly equal $v_{\mathrm{o}}^{z}$ (resp. $v_{\mathrm{w}}^{z}$ ) below (resp. above) the interface. The continuity of the total flux across the interface then yields

$$
-\frac{\kappa_{i}}{\mu_{\mathrm{o}}} \alpha_{i}=-\frac{\kappa_{i+1}}{\mu_{\mathrm{w}}} \alpha_{i+1} \text {. }
$$

Two additional equations are needed. They are obtained by fitting the nonhydrostatic water pressure with hydrostatic profiles at $z=b_{i+1}(\boldsymbol{x})$ and $z=\gamma_{i}(t, \boldsymbol{x})$. This leads respectively to the relations

$$
\begin{aligned}
\alpha_{i+1} H_{i+1}+\beta_{i+1} & =-\varrho_{\mathrm{w}} g b_{i}, \\
-\alpha_{i} h_{i}+\beta_{i} & =-\varrho_{\mathrm{w}} g b_{i}+\varrho g h_{i} .
\end{aligned}
$$

The system (29)-(32) admits a unique solution $\left(\alpha_{i}, \alpha_{i+1}, \beta_{i}, \beta_{i+1}\right)^{T}$ for any nonnegative $h_{i}$ and positive $H_{i+1}$, and

$$
\alpha_{i}=\frac{1}{h_{i}+\frac{\mu_{\mathrm{w}} \kappa_{i}}{\mu_{\mathrm{o}} \kappa_{i+1}} H_{i+1}}\left(p_{i+1}-p_{i}-\varrho g h_{i}\right) .
$$

Now, it remains to notice that since the flow is driven by buoyancy, the oil flux $S_{i}$ is always non-negative. When positive, it is given by

$$
S_{i}(t, \boldsymbol{x})=-\frac{\kappa_{i}}{\mu_{\mathrm{o}}}\left(\partial_{z} p_{\mathrm{o}}\left(t, \boldsymbol{x}, b_{i}(\boldsymbol{x})^{-}\right)+\varrho_{\mathrm{o}} g\right)=-\frac{\kappa_{i}}{\mu_{\mathrm{o}}} \alpha_{i},
$$

where $\partial_{z} p_{\mathrm{o}}\left(t, \boldsymbol{x}, b_{i}(\boldsymbol{x})^{-}\right)$denotes the left derivative at $z=b_{i}$ of the piecewise affine function $z \mapsto p_{\mathrm{o}}(t, \boldsymbol{x}, z)$. If the formula (33) provides a negative value, then $S_{i}(t, \boldsymbol{x})$ is set to 0, leading to Formula (10).

\section{Finite Volume approximation}

3.1. A two-point flux approximation Finite Volume scheme. We propose now Finite Volume scheme to approximate the solutions to problem (8)-(13). The equation (9) is of degenerate parabolic-elliptic type. We propose to discretize it thanks to a two-point flux approximation with upstream mobility. Since we use a two-point flux approximation of the diffusive flux, the mesh has to fulfil a socalled orthogonality condition (see [27] or [26]). Details on the discretization of the domain are presented in the next section. We give pieces of numerical analysis, showing the existence of solutions to the scheme together with its stability. These mathematical results are given as evidences of the relevance of our approach.

3.1.1. Discretization of $\mathbb{R}_{+} \times \Omega$. Before discretizing the time domain, we focus on the spatial domain. Taking advantage of the structure (1) of $\Omega$, we mainly have to mesh $\mathcal{O}$. In what follows, $\mathcal{O}$ is assumed to be polygonal if $d=2$. We use standard notations from [27].

An admissible discretization of $\mathcal{O}$ is made of a family of convex polygonal control volumes (or cells) $\mathcal{T}$, a family of edges $\mathcal{E}$ and a family of cell centers $\left(\boldsymbol{x}_{K}\right)_{K \in \mathcal{T}}$. The boundary of the control volumes are made of edges. More precisely, for all 
$K \in \mathcal{T}$, there exists a subset $\mathcal{E}_{K}$ of $\mathcal{E}$ such that $\partial K=\bigcup_{\sigma \in \mathcal{E}_{K}} \bar{\sigma}$. Moreover, we assume that $\mathcal{E}=\bigcup_{K \in \mathcal{T}} \mathcal{E}_{K}$. We assume that the control volumes mesh the whole $d$-dimensional domain $\mathcal{O}$, i.e., $\bigcup_{K \in \mathcal{T}} \bar{K}=\overline{\mathcal{O}}$, and that they are disjointed, i.e., $K \cap L=\emptyset$ if $K, L \in \mathcal{T}$ with $K \neq L$. More precisely, if $d=2$, given two distinct control volumes $K$ and $L$ in $\mathcal{T}$, either the either 1-dimensional Lebesgue measure of $\bar{K} \cap \bar{L}$ is equal to 0 , or there exists $\sigma \in \mathcal{E}$ such that $\bar{K} \cap \bar{L}=\bar{\sigma}$. If $d=1$, then the edges reduce to points and $\bar{K} \cap \bar{L}$ is either empty or it reduces to some $\sigma \in \mathcal{E}$. Finally, given two neighbouring cells $K$ and $L$ sharing an edge $\sigma$, then we assume that the straight line joining $\boldsymbol{x}_{K}$ to $\boldsymbol{x}_{L}$ is orthogonal to $\sigma$ and oriented in the same sense as the normal to $\sigma$ outward w.r.t. $K$. Finally, we decompose the set $\mathcal{E}$ of the edges into boundary edges $\mathcal{E}_{\text {ext }}=\{\sigma \in \mathcal{E} \mid \sigma \subset \partial \mathcal{O}\}$ and internal edges $\mathcal{E}_{\text {int }}=\{\sigma \in \mathcal{E} \mid \bar{\sigma}=\bar{K} \cap \bar{L}$ for some $K, L \in \mathcal{T}\}$. An internal edge $\sigma \in \mathcal{E}_{\text {int }}$ between two cells $K$ and $L$ is denoted by $K \mid L$.

For all $K \in \mathcal{T}$, we denote by $m_{K}$ the $d$-dimensional Lebesgue measure of $K$, while $m_{\sigma}$ denotes the $(d-1)$-dimensional Lebesgue measure of the edge $\sigma \in \mathcal{E}$. For $\sigma=K \mid L$, we denote the transmissivity of $\sigma$ by $\tau_{\sigma}=\frac{m_{\sigma}}{\left|\boldsymbol{x}_{K}-\boldsymbol{x}_{L}\right|}$.

For all $K \in \mathcal{T}$ and all $i \in\{0, \ldots, I\}$, we denote by $b_{i, K}=\frac{1}{m_{K}} \int_{K} b_{i}(\boldsymbol{x}) \mathrm{d} \boldsymbol{x}$, and by $H_{i, K}=b_{i, K}-b_{i-1, K}$ for $i \in\{1, \ldots, I\}$ the thickness of the $i^{\text {th }}$ layer in the column corresponding to the cell $K$.

Concerning time, we consider an increasing sequence $\left(t_{n}\right)_{n \geq 0}$ with $t_{0}=0$ and $\lim _{n \rightarrow \infty} t_{n}=+\infty$. We denote by $\Delta t_{n}=t_{n}-t_{n-1}$ for $n \geq 1$.

3.1.2. A time implicit finite volume scheme. We will now present the discretization of the system (8)-(13).

Let us first discretize the initial condition (11) by

$$
h_{i, K}^{0}=\frac{1}{m_{K}} \int_{K} h_{i}^{0}(\boldsymbol{x}) \mathrm{d} \boldsymbol{x}, \quad K \in \mathcal{T}, 1 \leq i \leq I .
$$

At each time step $n \geq 1$, we seek one oil thickness and capillary pressure per cell and per geological layer $\left(h_{i, K}^{n}, p_{i, K}^{n}\right)_{i, K}$ that are linked by the discrete counterpart to (8), namely

$$
p_{i, K}^{n} \in \widetilde{\pi}_{i, K}\left(h_{i, K}^{n}\right),
$$

where

$$
\tilde{\pi}_{i, K}(h)= \begin{cases}\pi_{i} & \text { if } 0<h<H_{i, K}, \\ \left(-\infty, \pi_{i}\right] & \text { if } h=0 \\ {\left[\pi_{i},+\infty\right)} & \text { if } h=H_{i, K} .\end{cases}
$$

They have to solve the discrete version of (9), that is

$$
\begin{aligned}
& \phi_{i} \frac{h_{i, K}^{n}-h_{i, K}^{n-1}}{\Delta t_{n}} m_{K} \\
& +\sum_{\sigma=K \mid L} \tau_{\sigma} \frac{\kappa_{i}}{\mu_{\mathrm{o}}} h_{i, \sigma}^{n}\left(p_{i, K}^{n}-p_{i, L}^{n}+\varrho g\left(h_{i, K}^{n}-h_{i, L}^{n}-b_{i, K}+b_{i, L}\right)\right) \\
& =\left(S_{i-1, K}^{n}-S_{i, K}^{n}\right) m_{K} .
\end{aligned}
$$


In (37), we used upwinding for the mobility: for all $\sigma=K \mid L \in \mathcal{E}_{\text {int }}$,

$$
h_{i, \sigma}^{n}= \begin{cases}h_{i, K}^{n} & \text { if } p_{i, K}^{n}+\varrho g\left(h_{i, K}^{n}-b_{i, K}\right) \geq p_{i, L}^{n}+\varrho g\left(h_{i, L}^{n}-b_{i, L}\right), \\ h_{i, L}^{n} & \text { otherwise. }\end{cases}
$$

Note that there is no term corresponding to lateral fluxes in the second term of (37) as a consequence of (12).

Concerning the exchange terms between the layers, one sets

$$
S_{i, K}^{n}=\frac{\kappa_{i}}{\mu_{w} \frac{\kappa_{i}}{\kappa_{i+1}} H_{i+1, K}+\mu_{o} h_{i, K}^{n}}\left(\varrho g h_{i, K}^{n}-\left(p_{i+1, K}^{n}-p_{i, K}^{n}\right)\right)^{+}
$$

for $i \in\{1, \ldots I-1\}$ and $K \in \mathcal{T}$ in accordance with (10), while

$$
S_{0, K}^{n}=S_{I, K}^{n}=0, \quad K \in \mathcal{T},
$$

as suggested by (13).

Given a solution $\left(h_{i, K}^{n}, p_{i, K}^{n}\right)_{i, K}$ to the scheme (35)-(40) - the existence of such a solution is the purpose of Theorem $3.3-$, we define $\boldsymbol{h}^{n}=\left(h_{i, K}^{n}\right)_{i, K}$ and $\boldsymbol{p}^{n}=$ $\left(p_{i, K}^{n}\right)_{i, K}$. We also reconstruct a piecewise constant in time and space approximate solution $\left(\boldsymbol{h}_{\mathcal{D}}, \boldsymbol{p}_{\mathcal{D}}\right)$ defined by

$$
h_{i, \mathcal{D}}^{n}(\boldsymbol{x})=h_{i, K}^{n} \text { and } p_{i, \mathcal{D}}^{n}(\boldsymbol{x})=p_{i, K}^{n} \text { if } \boldsymbol{x} \in K,
$$

and then by $\boldsymbol{h}_{\mathcal{D}}^{n}=\left(h_{i, \mathcal{D}}^{n}\right)_{1 \leq i \leq I}$ and $\boldsymbol{p}_{\mathcal{D}}^{n}=\left(p_{i, \mathcal{D}}^{n}\right)_{1 \leq i \leq I}$.

3.2. Auxiliary pressure and parametrization. Solving directly the non-linear system (35)-(40) may be difficult. One cannot directly use Newton's method because of the degeneracy of the graphs $\widetilde{\pi}_{i, K}$. One solution to overpass this difficulty is to use variable switch [22] between $\boldsymbol{h}^{n}$ and $\boldsymbol{p}^{n}$. The formalism we adopt here is inspired from $[9,11]$. It can be seen as a generalization of the variable switch even though its practical implementation is rather different.

Given $K \in \mathcal{T}$ and $i \in\{1, \ldots, N\}$, the monotone graph $\widetilde{\pi}_{i, K}$ defined by (36) can be parametrized by two non-decreasing and Lipschitz continuous functions $\bar{h}_{i, K}$ and $\bar{p}_{i, K}$ in the sense that

$$
p \in \tilde{\pi}_{i, K}(h) \text { iff there exists } u \in \mathbb{R} \text { s.t. } h=\bar{h}_{i, K}(u) \text { and } p=\bar{p}_{i, K}(u) .
$$

There are infinitely many functions $\bar{h}_{i, K}$ and $\bar{p}_{i, K}$ satisfying (42). One way to construct such functions is to use curvilinear coordinates along the graph $\widetilde{\pi}_{i, K}$. In what follows, we use the definitions

$$
\begin{aligned}
& \bar{h}_{i, K}(u)= \begin{cases}0 & \text { if } u \leq \pi_{i}, \\
\frac{1}{\varrho g}\left(u-\pi_{i}\right) & \text { if } u \in\left[\pi_{i}, \pi_{i}+\varrho g H_{i, K}\right], \\
H_{i, K} & \text { if } u \geq \pi_{i}+\varrho g H_{i, K},\end{cases} \\
& \bar{p}_{i, K}(u)= \begin{cases}u & \text { if } u \leq \pi_{i}, \\
\pi_{i} & \text { if } u \in\left[\pi_{i}, \pi_{i}+\varrho g H_{i, K}\right], \\
u-\varrho g H_{i, K} & \text { if } u \geq \pi_{i}+\varrho g H_{i, K} .\end{cases}
\end{aligned}
$$

so that

$$
\varrho g \bar{h}_{i, K}(u)+\bar{p}_{i, K}(u)=u, \quad \forall u \in \mathbb{R} .
$$


With this particular choice, the quantity $u_{i, K}^{n}$ such that $h_{i, K}^{n}=\bar{h}_{i, K}\left(u_{i, K}^{n}\right)$ and $p_{i, K}^{n}=\bar{p}_{i, K}\left(u_{i, K}^{n}\right)$ can be interpreted as the capillary pressure just below the interface $b_{i, K}^{n}$. We depict functions $\bar{h}_{i, K}$ and $\bar{p}_{i, K}$ defined by (43) corresponding to some graph $\widetilde{\pi}_{i, K}$ on Figure 3.
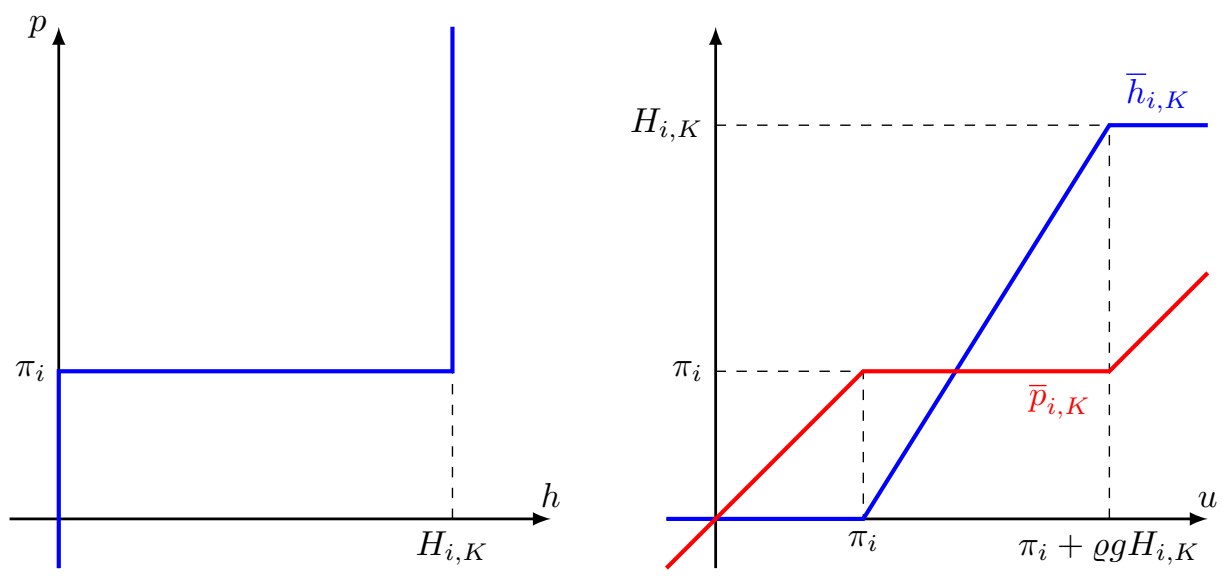

Figure 3. The maximal monotone graph $\tilde{\pi}_{i, K}$ (left) can be parametrized in the sense of (42) by the functions $\bar{h}_{i, K}$ and $\bar{p}_{i, K}$ defined by (43) (right).

Given $\boldsymbol{h}^{n-1}$ such that $0 \leq h_{i, K}^{n-1} \leq H_{i, K}$, the numerical scheme then amounts to find $\boldsymbol{u}^{n}=\left(u_{i, K}^{n}\right)_{i, K}$ such that, for all $i \in\{1, \ldots, I\}$ and all $K \in \mathcal{T}$, one has

$$
\begin{aligned}
& \phi_{i} \frac{\bar{h}_{i, K}\left(u_{i, K}^{n}\right)-h_{i, K}^{n-1}}{\Delta t_{n}} m_{K} \\
& \quad+\sum_{\sigma=K \mid L} \tau_{\sigma} \frac{\kappa_{i}}{\mu_{\mathrm{o}}} h_{i, \sigma}^{n}\left(u_{i, K}^{n}-u_{i, L}^{n}-\varrho g\left(b_{i, K}-b_{i, L}\right)\right)=\left(S_{i-1, K}^{n}-S_{i, K}^{n}\right) m_{K},
\end{aligned}
$$

where, thanks to (44), the upstream mobility can be expressed as

$$
h_{i, \sigma}^{n}= \begin{cases}h_{i, K}\left(u_{i, K}^{n}\right) & \text { if } u_{i, K}^{n}-\varrho g b_{i, K} \geq u_{i, L}^{n}-\varrho g b_{i, L}, \\ h_{i, L}\left(u_{i, L}^{n}\right) & \text { otherwise. }\end{cases}
$$

The interlayer fluxes $S_{i, K}^{n}$ are discretized by

$$
\begin{aligned}
S_{i, K}^{n} & =\bar{S}_{i, K}\left(u_{i, K}^{n}, u_{i+1, K}^{n}\right) \\
& =\frac{\kappa_{i}}{\mu_{w} \frac{\kappa_{i+1}}{\kappa_{i}} H_{i+1, K}+\mu_{o} h_{i, K}\left(u_{i, K}^{n}\right)}\left(u_{i, K}^{n}-\bar{p}_{i+1, K}\left(u_{i+1, K}^{n}\right)\right)^{+}
\end{aligned}
$$

if $i \in\{1, \ldots, I-1\}$, whereas we keep (40) for $i=0$ or $i=I$. Once $\boldsymbol{u}^{n}$ has been computed, one can recover the physical quantities $\boldsymbol{h}^{n}$ and $\boldsymbol{p}^{n}$ by setting

$$
h_{i, K}^{n}=\bar{h}_{i, K}\left(u_{i, K}^{n}\right) \quad \text { and } \quad p_{i, K}^{n}=\bar{p}_{i, K}\left(u_{i, K}^{n}\right), \quad K \in \mathcal{T}, i \in\{1, \ldots, I\} .
$$

In particular, the solution $\left(\boldsymbol{h}^{n}, \boldsymbol{p}^{n}\right) \in\left(\mathbb{R}^{I \times \# \mathcal{T}}\right)^{2}$ is fully characterized by the knowledge of $\boldsymbol{u}^{n} \in \mathbb{R}^{I \times \# \mathcal{T}}$. 
3.3. Stability analysis and existence of a discrete solution. The main goal of this section is to show that the proposed scheme (45)-(48) is stable and that it admits (at least) one solution $\boldsymbol{u}^{n}$ at each time step $n \geq 1$.

Our first lemma concerns the conservation of mass. It is a straightforward consequence of the conservativity of the scheme and of the definition (34) of the initial data. Its proof is left to the reader.

Lemma 3.1 (Conservation of the oil mass). Let $\boldsymbol{u}^{n}$ be a solution to the scheme (45)(47) and let $\boldsymbol{h}^{n}$ be given by (48), then

$$
\sum_{i=1}^{I} \sum_{K \in \mathcal{T}} m_{K} h_{i, K}^{n}=\sum_{i=1}^{I} \sum_{K \in \mathcal{T}} m_{K} h_{i, K}^{n-1}=\sum_{i=1}^{I} \int_{\mathcal{O}} h_{i}^{0}(\boldsymbol{x}) \mathrm{d} \boldsymbol{x}, \quad \forall n \geq 1 .
$$

3.3.1. Lack of uniqueness of the discrete solution $\boldsymbol{u}^{n}$. Before dealing with the existence of a discrete solution $\boldsymbol{u}^{n}$, let us briefly discuss about the lack of uniqueness.

Assume that $u_{i, K}^{n} \leq \pi_{i}$ for all $i$ and all $K$, so that $\boldsymbol{h}^{n}=\mathbf{0}$. Because of the conservation of mass, one has necessarily $\boldsymbol{h}^{n-1}=\mathbf{0}$ too. Therefore, the two first terms in (45) vanish and $\boldsymbol{u}^{n}$ is a solution as soon as $S_{i, K}\left(u_{i, K}^{n}, u_{i+1, K}^{n}\right)=0$ for all $i \in\{1, \ldots, I-1\}$. This condition is fulfilled as soon as $u_{i, K}^{n} \leq u_{i+1, K}^{n}$ for all $K \in \mathcal{T}$ and all $i \in\{1, \ldots, I-1\}$, so that it is easy to construct various solutions $\boldsymbol{u}^{n}$ corresponding to the trivial configuration $\boldsymbol{h}^{n}=\mathbf{0}$.

Similarly, one can construct many solutions corresponding to the case $\boldsymbol{h}^{n}=$ $\boldsymbol{h}^{n-1}=\boldsymbol{H}=\left(H_{i, K}\right)_{i, K}$, requiring that $u_{i, K}^{n} \geq \pi_{i}+\varrho g H_{i, K}$. The horizontal fluxes vanish as soon as

$$
u_{i, K}^{n}-\varrho g b_{i, K}=u_{i, L}^{n}-\varrho g b_{i, L}=C_{i}^{n}, \quad \forall(K, L) \in \mathcal{T}^{2} .
$$

The interlayer vertical fluxes $\left(S_{i, K}^{n}\right)_{i, K}$ vanish if

$$
u_{i, K}^{n} \leq \bar{p}_{i+1, K}\left(u_{i+1, K}^{n}\right)=u_{i+1, K}^{n}-\varrho g H_{i+1, K}, \quad K \in \mathcal{T}, 1 \leq i \leq I-1 .
$$

Here again, it is possible to construct several solutions $\boldsymbol{u}^{n}$ corresponding to the steady profile $\boldsymbol{h}^{n}=\boldsymbol{H}$.

3.3.2. Existence of a discrete solution. Our proof for the existence of a discrete solution is based on a topological degree argument [38, 21]. But in view of the degeneracy pointed out in Section 3.3.1, we have first add some stabilization terms to the scheme. To this end, define $b_{\star}=\min _{K} b_{0, K}$ and

$$
\pi_{\star}=\min _{1 \leq i \leq I} \pi_{i}, \quad \text { and } \quad u_{i, K}^{\star}=\max _{1 \leq j \leq I} \pi_{j}+\varrho g\left(b_{i, K}-b_{\star}\right) .
$$

The stabilized scheme is obtained by replacing (45) by

$$
\begin{aligned}
& \phi_{i} \frac{\bar{h}_{i, K}\left(u_{i, K}^{n}\right)-h_{i, K}^{n-1}}{\Delta t_{n}} m_{K}-\left(\pi_{\star}-u_{i, K}^{n}\right)^{+}+\left(u_{i, K}^{n}-u_{i, K}^{\star}\right)^{+} \\
& \quad+\sum_{\sigma=K \mid L} \tau_{\sigma} \frac{\kappa_{i}}{\mu_{\mathrm{o}}} h_{i, \sigma}^{n}\left(u_{i, K}^{n}-u_{i, L}^{n}-\varrho g\left(b_{i, K}-b_{i, L}\right)\right)=\left(S_{i-1, K}^{n}-S_{i, K}^{n}\right) m_{K}
\end{aligned}
$$

The following lemma shows the solutions to the stabilized scheme (49) are also solution to the original scheme (45). Notice that any solution $\boldsymbol{u}^{n}$ to the stabilized scheme is such that $\pi_{\star} \leq u_{i, K}^{n} \leq u_{i, K}^{\star}$. 
Lemma 3.2 (Uniform estimate on $\boldsymbol{u}^{n}$ ). Let $\boldsymbol{u}^{n}$ be a solution to (49), (46) and (47), then

$$
\pi_{\star} \leq u_{i, K}^{n} \leq u_{i, K}^{\star}, \quad K \in \mathcal{T}, 1 \leq i \leq I
$$

Proof. Let us first focus on the lower bound. Let $i_{0}, K_{0}$ be such that $u_{i_{0}, K_{0}}^{n}=$ $\min _{i, K} u_{i, K}^{n}$, and assume for contradiction that $u_{i_{0}, K_{0}}^{n}<\pi_{\star}$. Since $u_{i_{0}, K_{0}}^{n} \leq \pi_{i+1}$, it follows from the definitions (43b) and (47) that $S_{i_{0}, K_{0}}^{n}=0$. Moreover, since $h_{i_{0}, K_{0}}^{n}=0$, it follows from the upwind choice (46) of the mobility that

$$
\sum_{\sigma=K_{0} \mid L} \tau_{\sigma} \frac{\kappa_{i_{0}}}{\mu_{\mathrm{o}}} h_{i_{0}, \sigma}^{n}\left(u_{i_{0}, K_{0}}^{n}-u_{i_{0}, L}^{n}-\varrho g\left(b_{i_{0}, K_{0}}-b_{i_{0}, L}\right)\right) \leq 0 .
$$

Therefore, the left-hand side of (49) is negative because of the stabilization term, whereas the right-hand side is nonnegative, providing the expected contradiction.

Let us now establish the upper bound with a similar strategy. To this end, assume that

$$
i_{1}=\min \left\{i \in\{1, \ldots, I\} \mid \text { there exists } u_{i, K}^{n}>u_{i, K}^{\star}\right\}
$$

is well defined, i.e., the set on which we minimize is not empty. Let $K_{1}$ be such that

$$
u_{i_{1}, K_{1}}^{n}-\varrho g b_{i_{1}, K_{1}} \geq u_{i_{1}, L}^{n}-\varrho g b_{i_{1}, L}, \quad \forall L \in \mathcal{T} .
$$

This implies in particular, $u_{i_{1}, K_{1}}^{n}>u_{i_{1}, K_{1}}^{\star}$, so that

$$
u_{i_{1}, K_{1}}^{n}-\varrho g\left(b_{i_{1}, K_{1}}-b_{\star}\right)>\max _{j} \pi_{j} \geq u_{i_{1}-1, K_{1}}^{n}-\varrho g\left(b_{i_{1}-1, K_{1}}-b_{\star}\right)
$$

due to the definition of $i_{1}$. This leads to

$$
\bar{p}_{i_{1}, K_{1}}\left(u_{i_{1}, K_{1}}^{n}\right)=u_{i_{1}, K_{1}}^{n}-\varrho g H_{i_{1}, K_{1}}>u_{i_{1}-1, K_{1}}^{n},
$$

hence $S_{i_{1}-1, K_{1}}^{n}=0$. Since $b_{i_{1}, K_{1}} \geq b_{\star}+H_{i_{1}, K_{1}}$, we also deduce from (51) that $h_{i_{1}, K_{1}}^{n}=H_{i_{1}, K_{1}}$. Therefore, the left-hand side of (49) for $i=i_{1}$ and $K=K_{1}$ is positive because of the stabilization term, while the right-hand side is nonpositive. We get a contradiction, which implies that the minimization set in (50) is empty. Therefore, $u_{i, K}^{n} \leq u_{i, K}^{\star}$ for all $i \in\{1, \ldots, I\}$ and all $K \in \mathcal{T}$.

Theorem 3.3 (Existence of a solution to the scheme). There exists at least one solution to the scheme (45)-(47) complemented with (40) satisfying

$$
\pi_{\star} \leq u_{i, K}^{n} \leq u_{i, K}^{\star}, \quad \forall i \in\{1, \ldots, I\}, \forall K \in \mathcal{T} .
$$

Proof. The proof relies on a topological degree argument [38, 21]. Define the functional

$$
\mathcal{F}^{n}:\left\{\begin{array}{l}
\mathbb{R}^{I \times \# \mathcal{T}} \times[0,1] \rightarrow \mathbb{R}^{I \times \# \mathcal{T}} \\
(\boldsymbol{u}, \lambda) \mapsto\left(\mathcal{F}_{i, K}^{n}(\boldsymbol{u}, \lambda)\right)_{i, K}
\end{array}\right.
$$

where

$$
\begin{aligned}
\mathcal{F}_{i, K}^{n}(\boldsymbol{u}, \lambda)= & \lambda \phi_{i} \frac{\bar{h}_{i, K}\left(u_{i, K}\right)-h_{i, K}^{n-1}}{\Delta t_{n}} m_{K} \\
& -\lambda\left(\pi_{\star}-u_{i, K}\right)^{+}+\lambda\left(u_{i, K}-u_{i, K}^{\star}\right)^{+}+(1-\lambda)\left(u_{i, K}-\pi_{i}\right) \\
& +\lambda \sum_{\sigma=K \mid L} \tau_{\sigma} \frac{\kappa_{i}}{\mu_{\mathrm{o}}} h_{i, \sigma}\left(u_{i, K}-u_{i, L}-\varrho g\left(b_{i, K}-b_{i, L}\right)\right) \\
& +\lambda\left(\bar{S}_{i, K}\left(u_{i, K}, u_{i+1, K}\right)-\bar{S}_{i-1, K}\left(u_{i-1, K}, u_{i, K}\right)\right) m_{K},
\end{aligned}
$$


where $h_{i, \sigma}$ is defined by (46) but with generic $u_{i, K}$ and $u_{i, L}$ instead of values $u_{i, K}^{n}$ and $u_{i, L}^{n}$ of a discrete solution. Because of the Lipschitz continuity of $\bar{h}_{i, K}$ and $\bar{p}_{i, K}$, the functional $\mathcal{F}^{n}$ is uniformly continuous w.r.t. its two arguments.

Define the relatively compact open set

$$
\mathcal{U}=\left\{\boldsymbol{u} \in \mathbb{R}^{I \times \# \mathcal{T}} \mid \pi_{\star}-1<u_{i, K}<u_{i, K}^{\star}+1\right\} .
$$

For $\lambda=0, \mathcal{F}^{n}(\cdot, 0)$ turns out to be linear, and the system $\mathcal{F}^{n}(\boldsymbol{u}, 0)=\mathbf{0}$ admits $\boldsymbol{u}^{(0)}=\left(\pi_{i}\right)_{i, K}$ as a unique solution. The topological degree corresponding to $\mathcal{F}^{n}(\cdot, 0)$ and $\mathcal{U}$ is equal to 1 since $\boldsymbol{u}^{(0)}$ belongs to $\mathcal{U}$. Then one can adapt the proof of Lemma 3.2 to show that any solution $\boldsymbol{u}^{(\lambda)}$ to the system $\mathcal{F}^{n}\left(\boldsymbol{u}^{(\lambda)}, \lambda\right)=\mathbf{0}$ for $\lambda \in(0,1]$ necessarily belongs to $\mathcal{U}$. Therefore, the topological degree corresponding to $\mathcal{F}^{n}(\cdot, \lambda)$ and $\mathcal{U}$ does not depend on $\lambda$. In particular, it is also equal to 1 for $\lambda=1$, ensuring the existence of at least one solution $\boldsymbol{u}^{(1)}=\boldsymbol{u}^{n}$ to the stabilized scheme (49). Since the stabilization terms vanish on $\mathcal{U}, \boldsymbol{u}^{n}$ is also a solution to the scheme (45).

3.3.3. Energy stability of the scheme. The goal of this section is to establish the following discrete counterpart of Proposition 1.2, namely the decay of the energy if the system is isolated. Here, the discrete energy is noting but the continuous energy $\mathfrak{E}$ defined by (14) applied to the approximate solution $\boldsymbol{h}_{\mathcal{D}}$ defined by (41).

Proposition 3.4. Let $\boldsymbol{u}^{n}$ be a solution to the scheme (45)-(47) complemented with the no-flux boundary conditions (40), then $\mathfrak{E}\left(\boldsymbol{h}_{\mathcal{D}}^{n}\right) \leq \mathfrak{E}\left(\boldsymbol{h}_{\mathcal{D}}^{n-1}\right)$.

Proof. The proof follows the line of the one of Proposition 1.2. Multiplying the scheme (45) by $\Delta t_{n}\left(u_{i, K}^{n}-\varrho g b_{i, K}\right)$ then summing over $K \in \mathcal{T}$ and $i \in\{1, \ldots, I\}$ yield

$$
A_{\mathcal{T}}^{n}+B_{\mathcal{T}}^{n}+C_{\mathcal{T}}^{n}=0
$$

where we have set

$$
\begin{aligned}
A_{\mathcal{T}}^{n} & =\sum_{i=1}^{I} \sum_{K \in \mathcal{T}} m_{K}\left(h_{i, K}^{n}-h_{i, K}^{n-1}\right)\left(u_{i, K}^{n}-\varrho g b_{i, K}\right), \\
B_{\mathcal{T}}^{n} & =\sum_{i=1}^{I} \Delta t_{n} \sum_{\substack{\sigma \in \mathcal{E}_{\text {int }} \\
\sigma=K \mid L}} \tau_{\sigma} \frac{h_{i, \sigma}^{n}}{\mu_{\mathrm{o}}}\left|u_{i, K}^{n}-u_{i, L}^{n}-\varrho g\left(b_{i, K}-b_{i, L}\right)\right|^{2}, \\
C_{\mathcal{T}}^{n} & =\sum_{i=1}^{I-1} \Delta t_{n} \sum_{K \in \mathcal{T}} m_{K} S_{i, K}^{n}\left(u_{i, K}^{n}-u_{i+1, K}^{n}-\varrho g\left(b_{i, K}-b_{i+1, K}\right)\right) .
\end{aligned}
$$

The term $B_{\mathcal{T}}^{n}$ is obviously non-negative since $h_{i, \sigma}^{n} \geq 0$. For the term $A_{\mathcal{T}^{n}}$, let us first remark that since $0 \leq h_{i, K}^{n} \leq H_{i, K}$, one can rewrite

$$
\mathfrak{E}\left(\boldsymbol{h}_{\mathcal{D}}^{n}\right)=\sum_{i=1}^{I} \sum_{K \in \mathcal{T}} m_{K} E_{i, K}\left(h_{i, K}^{n}\right)
$$

where $E_{i, K}$ is the convex functional defined by

$$
E_{i, K}(h)= \begin{cases}\pi_{i} h+\frac{1}{2} \varrho g\left(b_{i, K}-h\right)^{2} & \text { if } 0 \leq h \leq H_{i, K}, \\ +\infty & \text { otherwise. }\end{cases}
$$


Given $h \in\left[0, H_{i, K}\right]$, the subdifferential of $E_{i, K}$ at $h$ is given by

$$
\partial E_{i, K}(h)=\left\{p+\varrho g\left(h-b_{i, K}\right) \mid p \in \widetilde{\pi}_{i, K}(h)\right\} .
$$

Therefore, a simple convexity inequality provides

$$
A_{\mathcal{T}}^{n} \geq \mathfrak{E}\left(\boldsymbol{h}_{\mathcal{D}}^{n}\right)-\mathfrak{E}\left(\boldsymbol{h}_{\mathcal{D}}^{n-1}\right) .
$$

As in the continuous case, we decompose

$$
\begin{aligned}
C_{\mathcal{T}}^{n}=\sum_{i=1}^{I-1} \Delta t_{n} \sum_{K \in \mathcal{T}} m_{K} \frac{\kappa_{i}}{\mu_{w} \frac{\kappa_{i}}{\kappa_{i+1}} H_{i+1, K}+\mu_{o} h_{i, K}^{n}}\left(\left(u_{i, K}^{n}-p_{i+1, K}^{n}\right)^{+}\right)^{2} \\
+\sum_{i=1}^{I-1} \Delta t_{n} \sum_{K \in \mathcal{T}} m_{K} S_{i, K}^{n}\left(H_{i+1, K}-h_{i+1, K}^{n}\right) .
\end{aligned}
$$

Both contributions are non-negative, hence so does $C_{\mathcal{T}}^{n}$.

Remark 3.5 (steady states). It is worth noticing in the proof of Proposition 3.4 that the term $B_{\mathcal{T}}^{n}$ vanishes iff all the horizontal fluxes vanish, while the term $C_{\mathcal{T}}^{n}$ vanishes iff all the interlayer vertical fluxes vanish. Therefore, either $\boldsymbol{h}^{n}$ is steady, i.e., $\boldsymbol{h}^{n}=\boldsymbol{h}^{n-1}$, or the energy is strictly decreasing. The steady states thus correspond to the solutions whose horizontal and vertical fluxes are both identically equal to 0 .

Remark 3.6 (Towards a convergence proof for the scheme). The energy estimate of Proposition 3.4 provides some compactness on a family of approximate solutions based on piecewise constant reconstruction. Roughly speaking, the nonlinear discrete Aubin-Lions lemma [3] provides strong (relative) compactness in $L^{r}((0, T) \times \mathcal{O})$, $1 \leq r<+\infty$, for the piecewise constant reconstruction $h_{i, \mathcal{T}, \Delta t}$ built from the values $h_{i, K}^{n}$, as well as weak compactness on the approximate interface capillary pressure $p_{i, \mathcal{T}, \Delta t}$ in $L^{2}((0, T) \times \mathcal{O})$. Moreover, weak $L^{2}((0, T) \times \mathcal{O})^{d}$ compactness can be derived on approximate gradients of $p_{i, \mathcal{T}, \Delta t}$ and $h_{i, \mathcal{T}, \Delta t}^{3 / 2}$. These compactness properties are enough to pass to the limit in the scheme to recover (9). Moreover, [3, Proposition 1.4] shows that the monotone relation (8) holds at the limit. The only issue to rigorously establish the convergence of the scheme concerns the recovery of the interlayer flux (10) because of the positive part which is nonlinear applied to the only weakly convergent quantity $u_{i, \mathcal{T}, \Delta t}-p_{i+1, \mathcal{T}, \Delta t}$ in (39). However, numerical experiments show no oscillatory behaviour and almost everywhere convergence seems to hold true, as illustrated by Figure 9.

\section{Numerical Results}

Before presenting numerical outcomes in Sections 4.3 and 4.4, we provide some details in Section 4.1 on the effective resolution of the nonlinear system (45)-(48). The efficiency of the solver, in particular the ability of the iterative method for the resolution, is paramount since we are interested in the long-time behavior of the model. Then after having compared our reduced model with a full Darcy model, we give numerical evidences of the ability of the model to capture multiple steady states depending on the dynamics. 
4.1. About the practical resolution. The nonlinear system (45)-(48) can be rewritten in the compact form $\mathcal{G}^{n}\left(\boldsymbol{u}^{n}\right)=\mathbf{0}$ with $\mathcal{G}^{n}=\mathcal{F}^{n}(\cdot, 1)$, where $\mathcal{F}^{n}$ is defined by (52). The Jacobian matrix $\mathbb{J}(\boldsymbol{u})$ of $\mathcal{G}^{n}$ at $\boldsymbol{u} \in \mathbb{R}^{I \times \# \mathcal{T}}$ might be non-invertible as a consequence of the lack of uniqueness highlighted in Section 3.3.1. This prohibits the direct use of Newton's method. Instead, our approach relies on a quasi-Newton method with a regularised approximate Jacobian matrix $\mathbb{J}_{\epsilon}(\boldsymbol{u})$ defined by $\mathbb{J}_{\epsilon}(\boldsymbol{u})=$ $\mathbb{J}(\boldsymbol{u})+\epsilon \mathbb{I}$ which is invertible. Practically, we choose $\epsilon=10^{-4}$ in our simulations. Moreover, we know from Theorem 3.3 that there exists a solution $\boldsymbol{u}^{n} \geq \pi_{\star} \mathbf{1}$ to the scheme $\mathcal{G}^{n}\left(\boldsymbol{u}^{n}\right)=\mathbf{0}$. This suggests the following quasi-Newton/projection algorithm.

Given $\boldsymbol{u}^{n-1} \in \mathbb{R}^{I \times \# \mathcal{T}}$, we initialize the sequence $\left(\boldsymbol{u}^{n, k}\right)_{k \geq 0}$ by setting $\boldsymbol{u}^{n, 0}=$ $\boldsymbol{u}^{n-1}$. Assume that $\boldsymbol{u}^{n, k}$, then $\boldsymbol{u}^{n, k+1}$ is computed as follows.

Semi-smooth quasi-Newton step. We first compute the intermediate state $\boldsymbol{u}^{n, k+1 / 2}$ thanks to a quasi-Newton iteration:

$$
\boldsymbol{u}^{n, k+1 / 2}=\boldsymbol{u}^{n, k}+\boldsymbol{w}^{n, k}
$$

where $\boldsymbol{w}^{n, k}$ solves

$$
\mathbb{J}_{\epsilon}\left(\boldsymbol{u}^{n, k}\right) \boldsymbol{w}^{n, k}+\mathcal{G}^{n}\left(\boldsymbol{u}^{n, k}\right)=\mathbf{0}
$$

Since $\mathcal{G}$ is not smooth but merely piecewise smooth, we enforce Newton's iterations to stop at discontinuities of $\mathbb{J}_{\epsilon}$. For instance, if $u_{i, K}^{n, k}<\pi_{i}$ and $u_{i, K}^{n, k}+w_{i, K}^{n, k}>\pi_{i}$, then we set $u_{i, K}^{n+1 / 2, k}=\pi_{i}+\bar{\epsilon}$. The parameter $\bar{\epsilon}$ is very small (it is practically set to $\left.\bar{\epsilon}=10^{-10}\right)$ and is there to give a proper sense to $\mathbb{J}_{\epsilon}\left(\boldsymbol{u}^{n+1 / 2}\right)$.

Projection step. The intermediate state $\boldsymbol{u}^{n, k+1 / 2}$ is then projected on $\left\{\boldsymbol{u} \geq \pi_{\star} \mathbf{1}\right\}$ by

$$
u_{i, K}^{n, k+1}=\max \left(\pi_{\star}, u_{i, K}^{n, k+1 / 2}\right), \quad k \geq 0,1 \leq i \leq I, K \in \mathcal{T} .
$$

Stopping criterion and adaptive time stepping. As suggested by [9], our stopping criterion is based on the $\ell^{1}$ norm of the residual, namely $\left\|\mathcal{G}^{n}\left(\boldsymbol{u}^{n, k}\right)\right\|_{\ell^{1}} \leq 10^{-8}$. In order to increase the robustness of the resolution, we use a classical adaptive time stepping algorithm. If the loop over $k$ has not yet converged after $k_{\max }$ iterations, we reinitialize the procedure but with a modified system corresponding to a reduced time step divided by two w.r.t. the previous attempt. But is the look over $k$ converges with less that $k_{\max }$ iterations, then the time step for the time iteration $n+1$ is chosen as $\Delta t_{n+1}=1.1 \times \Delta t_{n}$. Because of the degeneracy of the problem, the convergence can be rather slow. Therefore, we have to accept a possibly rather large number of iteration for each time step. This led us to fix $k_{\max }=50$ in our simulations.

4.2. A zero-dimensional test case. The goal of this section is to validate the flux formula (10) by comparing it to the results obtained from a Darcean model. The physical dimension is set to one, so that our reduced model becomes 0-dimensional, i.e., $d=0$. All the length are expressed in meters, whereas time is expressed in seconds. We consider a porous medium $\Omega$ made of three layers

$$
\Omega_{1}=(0,1000 \mathrm{~m}), \quad \Omega_{2}=(1000 \mathrm{~m}, 1500 \mathrm{~m}), \quad \Omega_{3}=(1500 \mathrm{~m}, 2000 \mathrm{~m}),
$$

the interfaces being located at $b_{1}=1000 \mathrm{~m}$ and $b_{2}=1500 \mathrm{~m}$. 
Since water is assumed to be hydrostatic (2), the Darcean model under consideration boils down to some Richards equation of the form

$$
\phi_{i} \partial_{t} s+\partial_{z} v_{\mathrm{o}, i}=0, \quad \text { with } \quad v_{\mathrm{o}, i}=-\kappa_{i} \frac{k_{\mathrm{o}}(s)}{\mu_{\mathrm{o}}} \partial_{z}(p-\varrho g z) \quad \text { in } \mathbb{R}_{+} \times \Omega_{i} .
$$

The capillary pressure $p: \mathbb{R}_{+} \times \Omega \rightarrow \mathbb{R}$ is related to the saturation thanks to the monotone relation

$$
p(t, z) \in \check{\pi}_{i}(s(t, z)) \quad \text { for }(t, z) \in \mathbb{R}_{+} \times \Omega_{i},
$$

where $\check{\pi}_{i}$ are defined in (18). At the interface $b_{i}$, the continuity of the capillary pressure $p$ and of the fluxes $v_{\mathrm{o}, i}=v_{\mathrm{o}, i+1}$ are prescribed as in [16, 14]. The initial saturation profile is given by

$$
s(0, z)= \begin{cases}1 & \text { if } z<b_{1}, \\ 0 & \text { if } z>b_{1},\end{cases}
$$

corresponding to $h_{1}(0)=H_{1}$ and $h_{2}(0)=h_{3}(0)=0$ for the reduced model. The Darcean model (55)-(57) is complemented with no-flux boundary conditions at $z=0$ and $z=2000$, whereas we impose $S_{0}=S_{3} \equiv 0$ for the reduced model. The Darcean model is approximated thanks to a fully implicit finite volume scheme with upstream mobility and parametrization of the monotone relation (56). We refer to [5] for details on the numerical method.

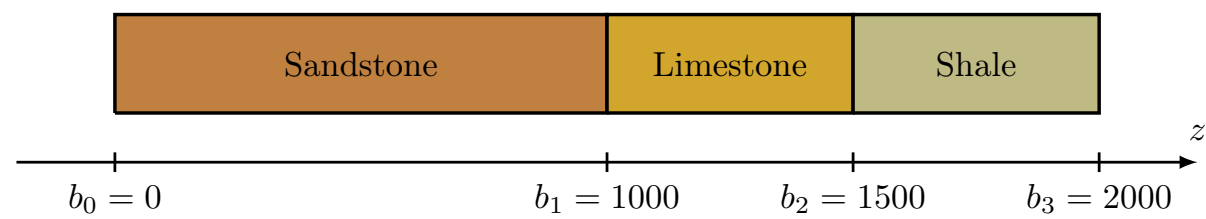

Figure 4. Schematic representation of the geometry for the 0dimensional test case.

The viscosity of each phases are given in centipoise (cPo) where $1 \mathrm{cPo}$ corresponds to $10^{-3} \mathrm{~Pa}$. The viscosity of the oil phase set to $\mu_{o}=11.78 \mathrm{cPo}$ while the viscosity of the water phase is set to $\mu_{w}=0.548 \mathrm{cPo}$. The permeability is given is mdarcy where 1 mdarcy corrresponds to $10^{-12} \mathrm{~m}^{2}$. The pressure is given in bar where 1 bar correspond to $10^{5} \mathrm{~Pa}$. The coefficient $\rho g$ is set to $\rho g=0.01962 \mathrm{bar} \mathrm{m}^{-1}$. The choices for the porosities, permeabilities, and capillary pressures depending on the rock type are reported in Table 1 . Two different choices of relative permeabilities are considered: a linear one $k_{\mathrm{o}}(s)=s$ and a quadratic one $k_{\mathrm{o}}(s)=s^{2}$. We plot in Figure 5 the flux across the interface $b_{1}$ as a function of time. Both choices for the relative permeabilities show a rather good (however not perfect) agreement with the interlayer flux $S_{1}(t)$ predicted by the reduced model. We also plot in Figure 6 the flux across $b_{2}$ together with $S_{2}$ as functions of time. There is a delay between the flux corresponding to the reduced model and the ones corresponding to the Darcean model. This is due to the time required for oil to cross $\Omega_{2}$ and reach the saturated region where $s=1$ located below $b_{2}$, which is neglected in the reduced model because of (28). The time required for oil to reach the saturated zone below $b_{2}$ naturally depends on the choice of the relative permeability $k_{\mathrm{o}}$. 


\begin{tabular}{|c||c|c|c|c|}
\hline Layer & Rock type & $\phi_{i}$ & $\kappa_{i}$ (mdarcy) & $\pi_{i}$ (bar) \\
\hline$\Omega_{3}$ & Shale & 0.2 & 2 & 20 \\
\hline$\Omega_{2}$ & Limestone & 0.3 & 10 & 15 \\
\hline$\Omega_{1}$ & Sandstone & 0.25 & 100 & 5 \\
\hline
\end{tabular}

TABLE 1. Properties of the geological layer

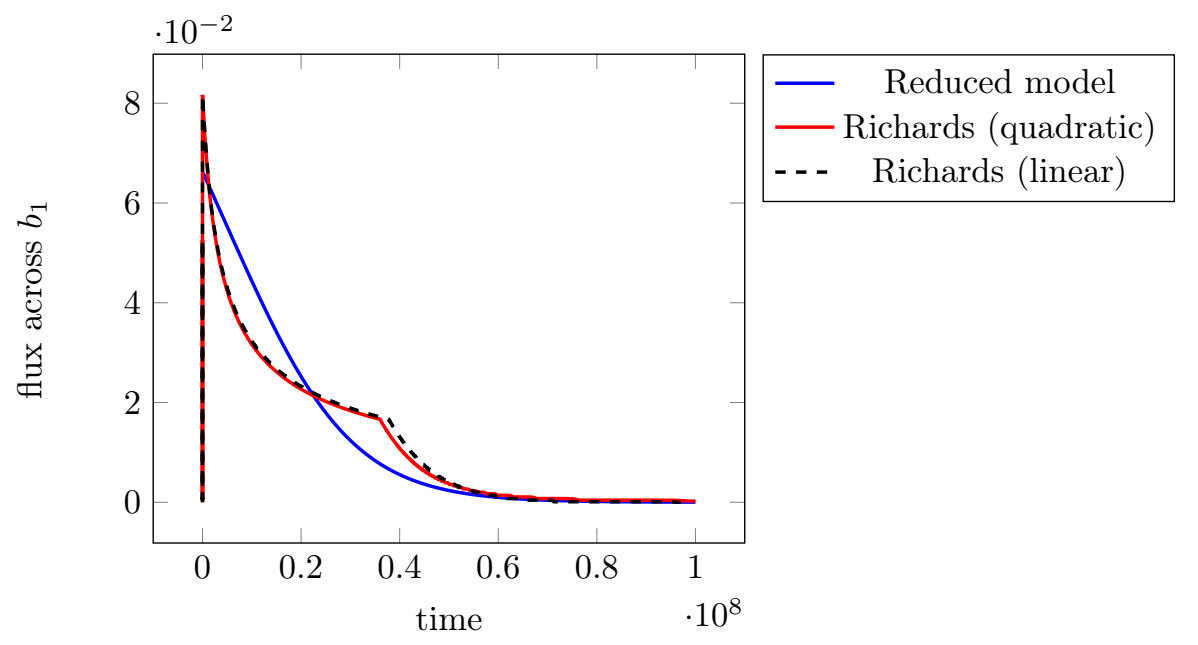

FiguRE 5. Flux across the first interface $b_{1}$ located at $z=1000 m$ starting from the bottom computed with the reduced model (blue solid line), with Richards equation with quadratic relative permeabilities (red solid line), and with Richards equation with linear relative permeabilities (dashed black line).

4.3. A one-dimensional test case. We propose a one-dimensional test case the goal of which is to highlight the importance of keeping track of the time variable. The geological basin is composed of a superposition of three vertical layers. More precisely the geological $\Omega_{i}$ is given by

$$
\Omega_{i}=\bigcup_{\boldsymbol{x} \in(0, L)}\{\boldsymbol{x}\} \times\left(b_{i-1}(\boldsymbol{x}), b_{i}(\boldsymbol{x})\right)
$$

where the functions $b_{i}, 0 \leq i \leq 3$, are given by

$$
\begin{aligned}
& b_{0}(\boldsymbol{x})=0, \\
& b_{1}(\boldsymbol{x})=\frac{H}{6}+\frac{H}{3 L}\left(\boldsymbol{x}+\frac{2 L}{3 \pi} \sin \left(\frac{3 \pi}{L} \boldsymbol{x}\right)\right), \\
& b_{2}(\boldsymbol{x})=\frac{5 H}{6}-\frac{2 H}{3 L^{2}}\left(\boldsymbol{x}-\frac{L}{2}\right)^{2}, \\
& b_{3}(\boldsymbol{x})=H .
\end{aligned}
$$




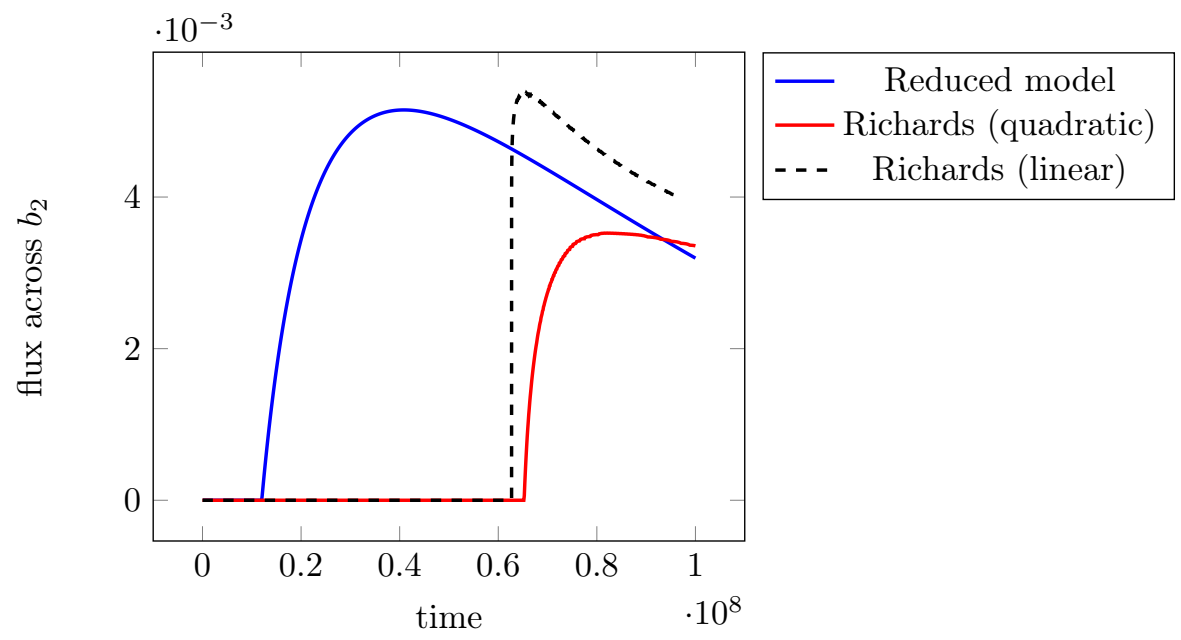

Figure 6. Flux across the first interface $b_{2}$ located at $z=1500 \mathrm{~m}$ starting from the bottom computed with the reduced model (blue solid line), with Richards equation with quadratic relative permeabilities (red solid line), and with Richards equation with linear relative permeabilities (dashed black line).

The length of the domain is set to $L=10^{4} \mathrm{~m}$. The geological properties of these vertical layers are given in Figure 4.3. The final time of the simulation is set to $10^{12} \mathrm{~s}$, which corresponds approximately to 31689 years.

\begin{tabular}{|c||c|c|c|c|}
\hline Layer & Rock type & $\phi_{i}$ & $\kappa_{i}$ (mdarcy) & $\pi_{i}$ (bar) \\
\hline$\Omega_{3}$ & Shale & 0.2 & 2 & 30 \\
\hline$\Omega_{2}$ & Limestone & 0.3 & 10 & 6.8 \\
\hline$\Omega_{1}$ & Sandstone & 0.25 & 100 & 5 \\
\hline
\end{tabular}

TABLE 2. Properties of the geological layer

There is no oil initially in the domain, namely

$$
h_{i}(0, \boldsymbol{x})=0, \quad 1 \leq i \leq 2, \boldsymbol{x} \in(0, L) .
$$

We assume no-flux boundary conditions on the lateral boundary as well as across the top layer, i.e., $S_{2}(t, \boldsymbol{x})=0$. Oil is injected at the bottom of the domain,

$$
S_{0}(t, \boldsymbol{x})=s_{0} 1_{0<t<t_{0}}(t) 1_{L_{1} \leq \boldsymbol{x} \leq L_{2}}(\boldsymbol{x}) \quad t \geq 0, \boldsymbol{x} \in(0, L),
$$

where the source is localized between $L_{1}=2500 \mathrm{~m}$ and $L_{2}=2600 \mathrm{~m}$. We investigate the long time behavior of the model for two different injection regimes:

- a fast injection regime where $s_{0}=0.01 \mathrm{~m} \mathrm{~s}^{-1}$ and $t_{0}=10^{6} \mathrm{~s} \simeq 11,57$ days.

- a slow injection regime where $s_{0}=2.10^{-7} \mathrm{~m} \mathrm{~s}^{-1}$ and $t_{0}=5.10^{10} \mathrm{~s} \simeq 1584$ years.

Note that the total quantity of oil at the final time in the domain is the same for both regimes since $\int_{0}^{T} S_{0}(t, \boldsymbol{x}) \mathrm{d} t$ takes the same values in both cases. Therefore, 
reduced models where the time evolution is neglected would compute the same equilibrium for both scenarios. We assume that

$$
S_{3}(t, \boldsymbol{x})=0, \quad t \geq 0, \boldsymbol{x} \in(0, L) .
$$

Remark 4.1 (Inhomogeneous inflow and outflow conditions). The inflow condition (59) is not covered by the numerical analysis developed in Section 3.3. Inhomogeneous inflow or outflow conditions cannot be handled in full generality since they can correspond to situations where the discrete system has no solution. This is for instance the case of $\int_{\mathcal{O}} S_{0}(t, \boldsymbol{x}) \mathrm{d} \boldsymbol{x}>0$ while $S_{N}(t, \cdot) \equiv 0$ and all the layers are fully saturated by oil, i.e. $h_{i} \equiv H_{i}$ for all $i$. However, the configuration chosen in this test case (and in the two-dimensional one presented in Section 4.4) leads to a well posed discrete system, as illustrated by the numerical results.

For the space discretization we consider a uniform discretization with 1200 cells. This source term is discretized by

$$
S_{0, K}^{n}=\frac{1}{m_{K} \Delta t_{n}} \int_{t^{n-1}}^{t^{n}} \int_{K} S_{0}(t, \boldsymbol{x}) \mathrm{d} \boldsymbol{x} \mathrm{d} t, \quad K \in \mathcal{T},
$$

The evolution of the interfaces between oil and water are represented on Figure 7 and Figure 8 for the fast and slow injection regimes respectively. Notice that the final steady state is different in both situations, as predicted in [48].

Although we were not able to prove theoretically the convergence of the scheme, cf. Remark 3.6, we have numerical evidences of its convergence. On Figure 9, we plot the relative $L^{1}$ error on the entrapped heights as a function of the number of cells. The test cases under consideration are the same as the one depicted on Figures 7 and 8, with either fast or slow injection of oil at the bottom of the computational domain. Since we do not have an exact solution for the problem, a reference solution $\boldsymbol{u}_{\text {ref }}^{N}$ at final time $t=10^{12} \mathrm{~s}$ is computed on a fine grid made of 6400 cells, from which we build a reference heights $h_{i, \mathcal{D}_{\text {ref }}}^{N}$ thanks to (41). Then the relative $L^{1}$ error is then given by

$$
\operatorname{Err}_{\mathcal{D}, 1}=\frac{\sum_{i=1}^{I}\left\|h_{i, \mathcal{D}}^{N}-h_{i, \mathcal{D}_{\text {ref }}}^{N}\right\|_{L^{1}(\mathcal{O})}}{\sum_{i=1}^{I}\left\|h_{i, \mathcal{D}_{\text {ref }}}^{N}\right\|_{L^{1}(\mathcal{O})}} .
$$

Figure 9 shows a first order convergence for both injection scenarios. The error is rather slow even thought the final time is extremely large. We interpret this remarkable long-time behaviour of the scheme as a consequence of its energy stability.

4.4. A two-dimensional test case. We propose a two-dimensional test case where the geological basin is composed of a superposition of two layers. More precisely the geometry of the geological layers $\Omega_{1}, \Omega_{2}$ is given by

$$
\Omega_{i}=\bigcup_{\boldsymbol{x} \in \mathcal{O}}\{\boldsymbol{x}\} \times\left(b_{i-1}(\boldsymbol{x}), b_{i}(\boldsymbol{x})\right), \quad i \in\{1,2\},
$$

where $\mathcal{O}=(0, L)^{2}$ with $L=10^{4} \mathrm{~m}$. The interfaces $\left(b_{i}\right)_{0 \leq i \leq 2}$ are given by $b_{0}(\boldsymbol{x})=0$,

$$
\begin{aligned}
& b_{1}(x, y)=400\left[\cos \left(\frac{5 x}{2 L}\right) \sin \left(\frac{2 y}{L}\right)+\frac{5}{3}\right], \\
& b_{2}(x, y)=400\left[\sin \left(\frac{2 x}{L}\right) \cos \left(\frac{5 y}{2 L}\right)+\frac{10}{3}\right] .
\end{aligned}
$$



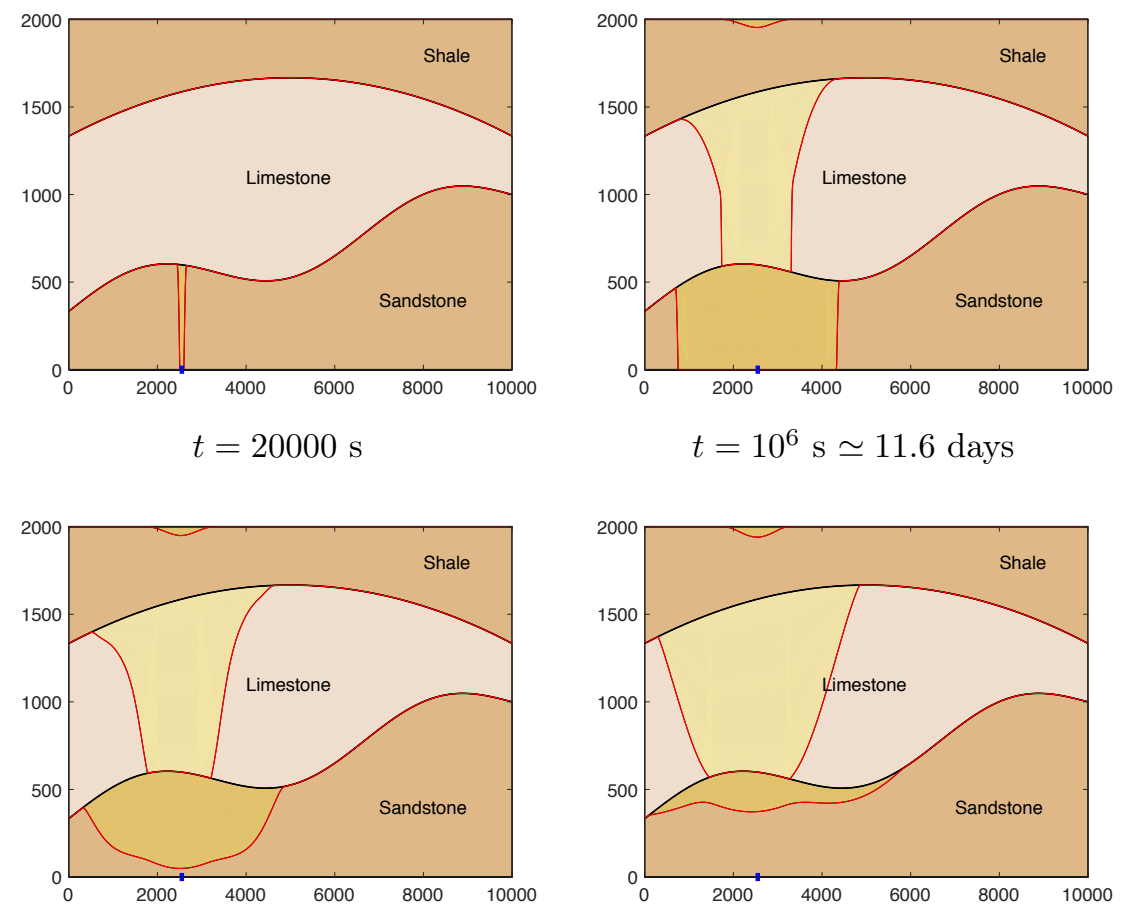

$t=5.10^{6} \mathrm{~s} \simeq 57.9$ days

$t=5.10^{7} \mathrm{~s} \simeq 579$ days
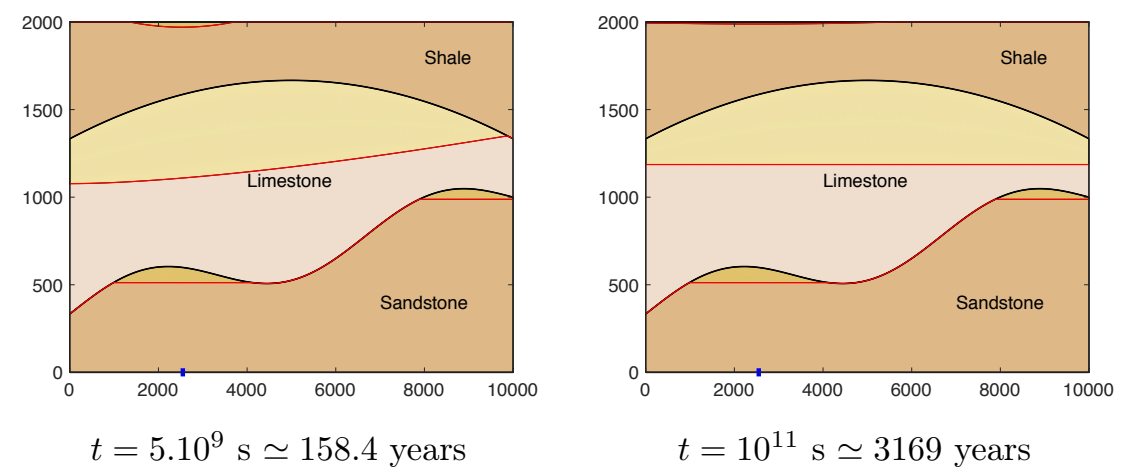

Figure 7. Time evolution in the fast injection regime.

The physical properties of the two different rocks are described in Table 4.4. The physical properties of the two fluids are chosen as in Section 4.3.

In each geological layer the thickness of the oil layer is initially set to zero, i.e.,

$$
h_{i}(0, \boldsymbol{x})=0, \quad 1 \leq i \leq 2, \boldsymbol{x} \in \mathcal{O},
$$

We assume no-flux boundary conditions across the lateral boundaries as well as across the roof, i.e., $S_{2} \equiv 0$. Concerning the source term, we consider again a fast and a slow injection regime. For the fast injection regime, oil is injected through a part $\omega=(600,2400)^{2}$ of the bottom of the domain at the rate of $5.10^{-5} \mathrm{~m} \mathrm{~s}^{-1}$ 

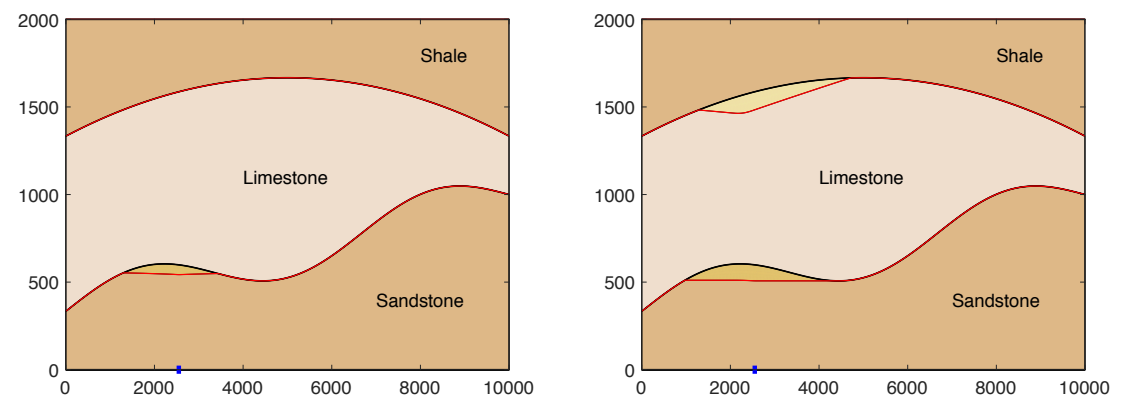

$$
t=5.10^{9} \mathrm{~s} \simeq 158.4 \text { years }
$$

$$
t=2.10^{10} \mathrm{~s} \simeq 633.8 \text { years }
$$
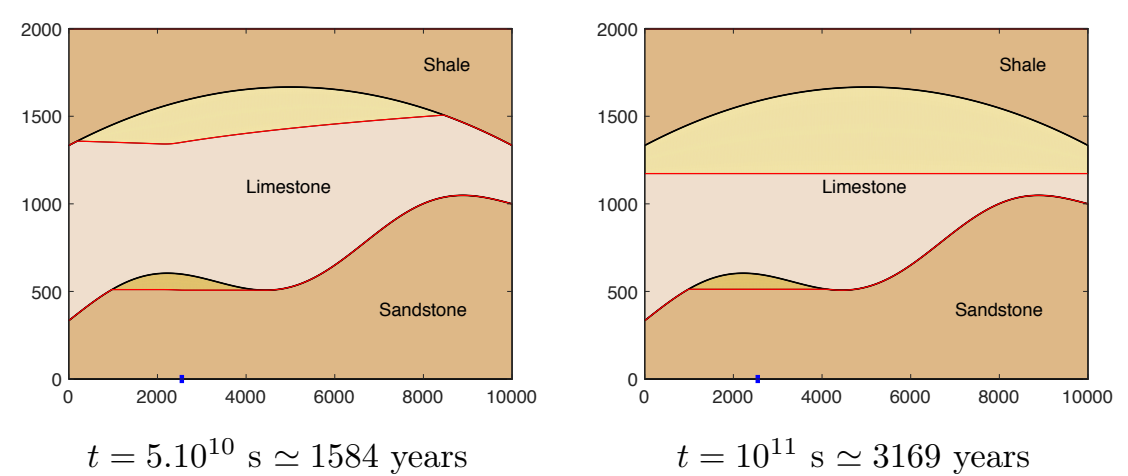

$t=5.10^{10} \mathrm{~s} \simeq 1584$ years

FiguRE 8. Time evolution in the slow injection regime.

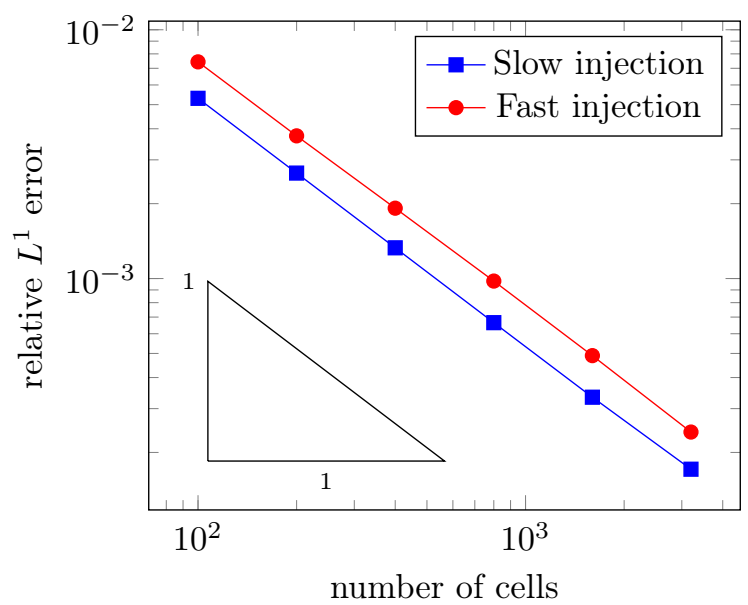

Figure 9. Relative $L^{1}$ error (62) with respect to the solution computed on a 6400 cell mesh at final time $t=10^{11} \mathrm{~s}$ for $1 \mathrm{D}$ settings.

between $t=0$ and $t=10^{7} \mathrm{~s} \simeq 115,74$ days, leading to the following formula:

$$
S_{0}(t, \boldsymbol{x})=5.10^{-5} \times 1_{\omega}(\boldsymbol{x}) \times 1_{\left(0,10^{7}\right)}(t), \quad \forall(t, \boldsymbol{x}) \in \mathbb{R}_{+} \times \mathcal{O}
$$




\begin{tabular}{|c||c|c|c|c|}
\hline Layer & Rock type & $\phi_{i}$ & $\kappa_{i}$ (mdarcy) & $\pi_{i}$ (bar) \\
\hline$\Omega_{2}$ & Limestone & 0.3 & 10 & 20 \\
\hline$\Omega_{1}$ & Sandstone & 0.25 & 100 & 12 \\
\hline
\end{tabular}

TABLE 3. Properties of the geological layer

The injection zone $\omega$ is highlighted in blue on Figure 10 .

The set $\mathcal{O}$ is discretized thanks to a $51 \times 51$ uniform cartesian grid. We use an adaptive time stepping strategy with time steps between $10^{8} \mathrm{~s}$ and $5.10^{10} \mathrm{~s}$.

The evolution along time of the heights of the interfaces is represented on Figure 10. We notice that It shows that oil invades the upper layer right above the source. This result should be compared to a slow injection regime where

$$
S_{0}(t, \boldsymbol{x})=5 \cdot 10^{-10} \times 1_{\omega}(\boldsymbol{x}) \times 1_{\left(0,10^{12}\right)}(t), \quad \forall(t, \boldsymbol{x}) \in \mathbb{R}_{+} \times \mathcal{O},
$$

for which the steady state is presented in Figure 11. For this slow injection regime, oil has enough time to creep into the dome located near $(x, y)=(0,8000)$ and to accumulate there. The total quantity of injected oil (which is the same in both regimes) is not sufficient to reach the critical height $\frac{\pi_{2}-\pi_{1}}{\rho g} \simeq 407.75 \mathrm{~m}$ and the whole oil phase remains therefore trapped in the lower layer.
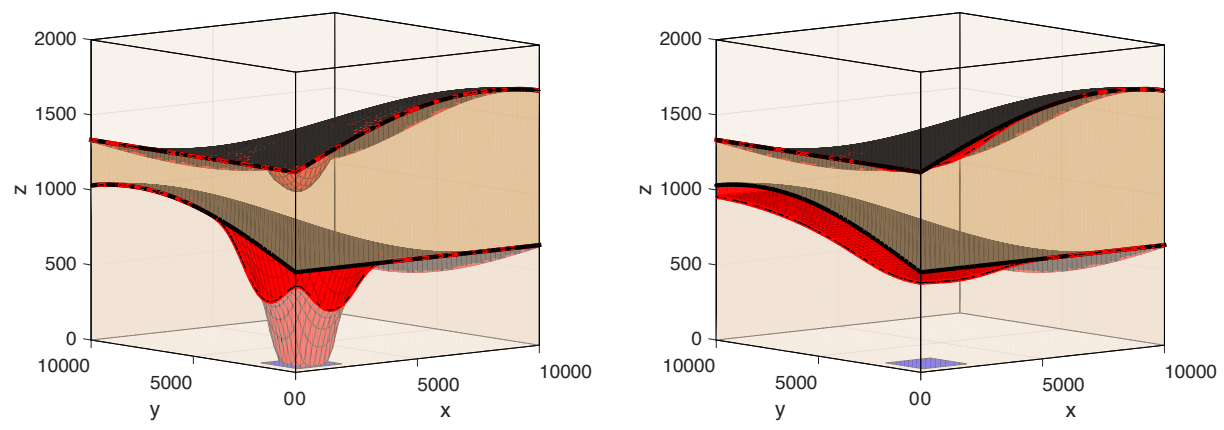

$t=10^{7} \mathrm{~s} \simeq 11.74$ days


$t=5.10^{9} \mathrm{~s} \simeq 158.44$ years

$t=10^{12} \mathrm{~s} \simeq 31689$ years

Figure 10. Evolution of the model in the two-dimensional setting for the fast injection regime. 

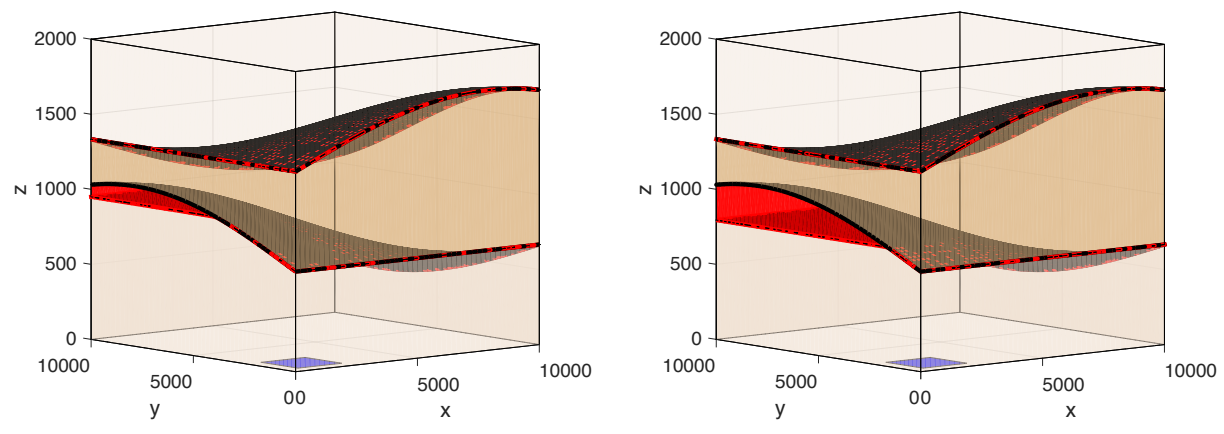

$t=2 \cdot 10^{11} s \simeq 6338$ years

$t=2.10^{12} s \simeq 63378$ years

FiguRE 11. Evolution of the model in the two-dimensional setting for the slow injection regime.

The oil thickness is compared along time to the height predicted by a simple raytracing algorithm on Figure 12. It gives an evidence that the slow injection regimes yields the same steady state as the one predicted by the ray-tracing algorithm. This is no longer true for the fast infection regime, for which about $15 \%$ of the oil quantity leaves the lower layer $\Omega_{1}$ to get trapped in the upper layer $\Omega_{2}$.

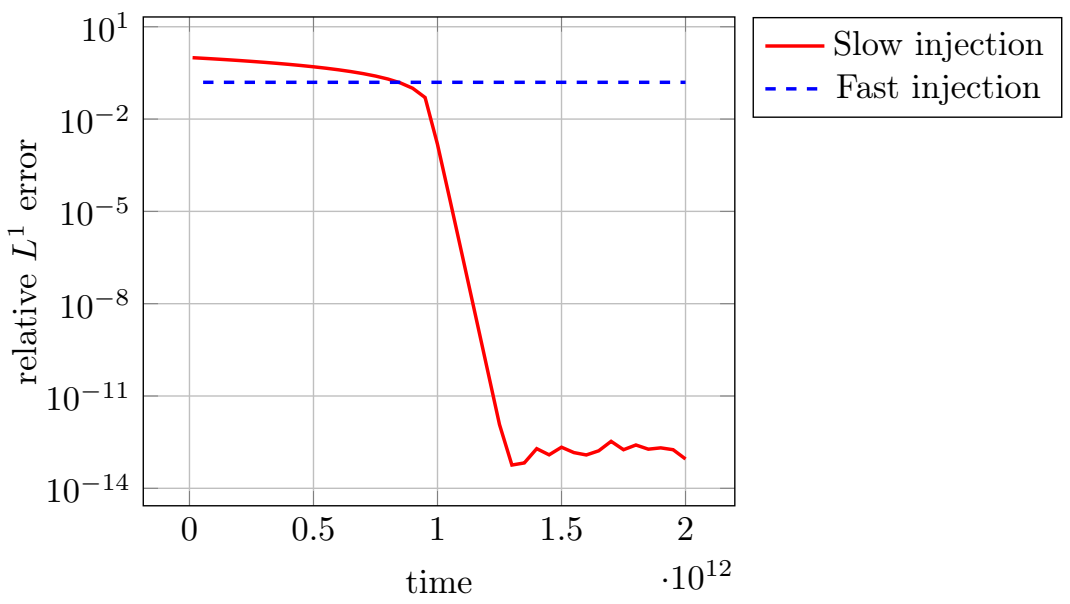

Figure 12. Evolution of the relative $L^{1}$ distance between the solution given by our model and the one given by the ray-tracing algorithm.

\section{Conclusion}

The derivation of a computationally efficient numerical model for oil migration in geological basins which is able to reproduce oil trapping and to capture different steady states depending on the dynamics of source term located at the bottom of the domain. The model keeps track of the time variable, in opposition to ray-tracing and invasion-percolation algorithms. Its lower complexity when compared to full 
Darcean models comes from dimensional reduction based on vertical integration and dimension reduction. The exchange terms between the layers are determined thanks to a one dimensional study relaxing the equilibrium assumption in the vertical direction. The resulting contribution, which shares common features with formerly proposed models while being different, allows to reproduce oil trapping. Moreover, our model inherits its stability from the full Darcean model it was derived from. This remarkable stability property is transposed to the fully discrete setting once our continuous model has been approximated by the mean of a fully implicit twopoint flux approximation finite volume scheme. The stability of the system also allows to show that the nonlinear system resulting from the scheme is well-posed. Our last contribution concerns the efficient computation of the solution to this nonlinear system. We take advantage of recent breakthroughs on the discretization of degenerate parabolic partial differential equations to propose a parametrization based cut-Newton method which appears to be extremely efficient. Our numerical model then allows for large time steps, making the computation of steady states possible.

This study involved contributions concerning the modeling, the numerical analysis and scientific computing. The interplay between these contributions allowed to obtain convincing preliminary numerical results. The validation of the model appears naturally as the following step. Comparison of our reduced model with Darcean models (which are much more demanding from a computational point of view) are still at a very early stage, but it already shows the need of incorporating a delay term in formula (28) to take into account the time needed for oil to migrate from one accumulation zone to another. This will be explored in a future work.

Acknowledgements. This work was partially supported by the Labex CEMPI (ANR-11-LABX-0007-01). The authors warmly thank the anonymous referees for their valuable comments and suggestions. The authors also thank Konstantin Brenner, Guillaume Enchéry and Roland Masson for the shared valuable discussions on the model.

\section{REFERENCES}

[1] A. Ait Hammou Oulhaj. Numerical analysis of a finite volume scheme for a seawater intrusion model with cross-diffusion in an unconfined aquifer. Numer. Methods Partial Differential Equations, 34(3):857-880, 2018.

[2] A. Ait Hammou Oulhaj, C. Cancès, C. Chainais-Hillairet, and P. Laurençot. Large time behavior of a two phase extension of the porous medium equation. Interfaces Free Bound., 21(2):199-229, 2019.

[3] B. Andreianov, C. Cancès, and A. Moussa. A nonlinear time compactness result and applications to discretization of degenerate parabolic-elliptic PDEs. J. Funct. Anal., 273(12):3633$3670,2017$.

[4] K. Aziz and A. Settari Petroleum Reservoir Simulation. Elsevier Applied Science Publishers, London, 1979.

[5] S. Bassetto, C. Cancès, G. Enchéry, and Q. H. Tran. Robust Newton solver based on variable switch for a finite volume discretization of Richards equation. Finite Volumes for Complex Applications IX - Methods, Theoretical Aspects, Examples. R. Klöfkorn, E. Keilegavlen, F. A. Radu \& J. Fuhrmann (ed.), Proceedings in Mathematics and Statistics 323, pp. 385-394, Springer [Cham], 2020.

[6] J. Bear. Hydraulics of groundwater. McGraw-Hill, Inc., New York, 1979.

[7] J. Bear and Y. Bachmat. Introduction to modeling of transport phenomena in porous media. Kluwer Academic Publishers, Dordrecht, The Netherlands, 1990.

[8] R. R. Berg. Capillary pressures in stratigraphic traps. AAPG bulletin, 59(6):939-956, 1975. 
[9] K. Brenner and C. Cancès. Improving Newton's method performance by parametrization: The case of the Richards equation. SIAM J. Numer. Anal., 55(4):1760-1785, 2017.

[10] K. Brenner, C. Cancès, and D. Hilhorst. Finite volume approximation for an immiscible two-phase flow in porous media with discontinuous capillary pressure. Comput. Geosci., 17(3):573-597, 2013.

[11] K. Brenner, M. Groza, L. Jeannin, R. Masson, and J. Pellerin. Immiscible two-phase darcy flow model accounting for vanishing and discontinuous capillary pressures: application to the flow in fractured porous media. Comput. Geosci., 21(5-6):1075-1094, 2017.

[12] H. Brézis. Opérateurs maximaux monotones et semi-groupes de contractions dans les espaces de Hilbert. North-Holland Publishing Co., Amsterdam-London; American Elsevier Publishing Co., Inc., New York, 1973.

[13] F. Buzzi, M. Lenzinger, and B. Schweizer. Interface conditions for degenerate two-phase flow equations in one space dimension. Analysis, 29:299-316, 2009.

[14] C. Cancès. Finite volume scheme for two-phase flow in heterogeneous porous media involving capillary pressure discontinuities. M2AN Math. Model. Numer. Anal., 43:973-1001, 2009.

[15] C. Cancès. On the effects of discontinuous capillarities for immiscible two-phase flows in porous media made of several rock-types. Netw. Heterog. Media, 5:635-647, 2010.

[16] C. Cancès, T. Gallouët, and A. Porretta. Two-phase flows involving capillary barriers in heterogeneous porous media. Interfaces Free Bound., 11(2):239-258, 2009.

[17] C. Cancès, T. O. Gallouët, and L. Monsaingeon. The gradient flow structure of immiscible incompressible two-phase flows in porous media. C. R. Acad. Sci. Paris Sér. I Math., 353:985989, 2015.

[18] C. Cancès, T. O. Gallouët, and L. Monsaingeon. Incompressible immiscible multiphase flows in porous media: a variational approach. Anal. PDE, 10(8):1845-1876, 2017.

[19] C. Cancès and M. Pierre. An existence result for multidimensional immiscible two-phase flows with discontinuous capillary pressure field. SIAM J. Math. Anal., 44(2):966-992, 2012.

[20] C. Choquet, M. M. Diédhiou, and C. Rosier. Derivation of a sharp-diffuse interfaces model for seawater intrusion in a free aquifer. SIAM J. of Appl. Math., 76(1):138-158, 2016.

[21] K. Deimling. Nonlinear functional analysis. Springer-Verlag, Berlin, 1985.

[22] H.-J. G. Diersch and P. Perrochet. On the primary variable switching technique for simulating unsaturated-saturated flows. Adv. Water Resour., 23(3):271-301, 1999.

[23] C. J. van Duijn, J. Molenaar, J. and M. J. de Neef. The effect of capillary forces on immiscible two-phase flows in heterogeneous porous media Transport Porous Med., 21:71-93, 1995.

[24] J. Dupuit. Études théoriques et pratiques sur le mouvement des eaux dans les canaux découverts et à travers les terrains perméables. Dunod, Paris, 1863.

[25] W. A. England, A. S. Mackenzie, D. M. Mann, and T. M. Quigley. The movement and entrapment of petroleum fluids in the subsurface. J. Geol. Soc., 144: 327-347, 1987.

[26] R. Eymard, T. Gallouët, C. Guichard, R. Herbin, and R. Masson. TP or not TP, that is the question. Comput. Geosci., 18:285-296, 2014.

[27] R. Eymard, T. Gallouët, and R. Herbin. Finite volume methods. Ciarlet, P. G. (ed.) et al., in Handbook of numerical analysis. North-Holland, Amsterdam, pp. 713-1020, 2000.

[28] R. Eymard, R. Herbin, and A. Michel. Mathematical study of a petroleum-engineering scheme. M2AN Math. Model. Numer. Anal., 37(6):937-972, 2003.

[29] M. J. Golding, J. A. Neufeld, M. A. Hesse, and Huppert. Two-phase gravity currents in porous media. J. Fluid Mech., 678:248-270, 2011.

[30] I. Gunn and A. W. Woods. On the flow of buoyant fluid injected into a confined, inclined aquifer. J. Fluid Mech., 672:109-129, 2011.

[31] M. A. Hesse, F. M. Jr Orr, and H. A. Tchelepi. Scaling analysis in the migration of $\mathrm{CO}_{2}$ in saline aquifers. J. Fluid Mech., 611:35-60, 2008.

[32] M. A. Hesse, F. M. Jr Orr, and H. A. Tchelepi. Gravity currents with residual trapping. J. Fluid Mech., 611:35-60, 2008.

[33] B. Hunt. Flow to a well in a multiaquifer system. Water Resour. Res., 21(11):1637-1641, 1985.

[34] H. E. Huppert and A. W. Woods. Gravity-driven flows in porous layers. J. Fluid Mech., 292:55-69, 1995.

[35] M. Jazar and R. Monneau. Derivation of seawater intrusion models by formal asymptotics. SIAM J. Appl. Math., 74(4):1152-1173, 2014. 
[36] R. Juanes, C. W. MacMinn, and M. L. Szulczewski. The footprint of the $\mathrm{CO}_{2}$ plume during carbon dioxide storage in saline aquifers: Storage efficiency for capillary trapping at the basin scale. Transp. Porous Med., 82:19-30, 2010.

[37] M. Kang, J. M. Nordbotten, F. Doster, and M. A. Celia Analytical solutions for two-phase subsurface flow to a leaky fault considering vertical flow effects and fault properties. Water Resour. Res., 50:3536-3552, 2014.

Analytical solutions for two-phase subsurface flow to a leaky fault considering vertical flow effects and fault properties. Water Resour. Res., 50:3536-3552, 2014.

[38] J. Leray and J. Schauder. Topologie et équations fonctionnelles. Ann. Sci. École Norm. Sup. (3), 51:45-78, 1934.

[39] R. J. McCann. A Convexity Principle for Interacting Gases Adv. Math., 128(1):153-179, 1997.

[40] Y. Masson and S. R. Pride. A fast algorithm for invasion percolation. Transp. Porous Med., 102:301-312, 2014.

[41] T. J. Murphy and N. J. Walkington. Control volume approximation of degenerate two-phase porous flows. SIAM J. Numer. Anal., 57(2):527-546, 2019.

[42] J. A. Neufeld, D. Vella, H. E. Huppert, and J. R. Lister. Leakage from gravity currents in a porous medium. Part 1. A localized sink. J. Fluid Mech. 666:391-413; 2011.

[43] J. A. Neufeld, D. Vella, H. E. Huppert, and J. R. Lister. Leakage from gravity currents in a porous medium. Part 2. A line sink. J. Fluid Mech. 666:414-427; 2011.

[44] T. D. Ngo, E. Mouche, and P. Audigane. Buoyant flow of $\mathrm{CO}_{2}$ through and around a semipermeable layer of finite extent. J. Fluid Mech., 809:553-584, 2016.

[45] J. M. Nordbotten and M. Celia. Similarity solutions for fluid injection into confined aquifers. J. Fluid. Mech., 561:307-327, 2006.

[46] J. M. Nordbotten and M. Celia. An improved analytical solution for interface upconing around a well. Water Resour. Res., 42(8):W08433, 2006.

[47] J. M. Nordbotten and M. Celia. Geological Storage of CO2: Modeling Approaches for Largescale Simulation. John Wiley \& Sons, New Jersey, 2012.

[48] S. Pegaz-Fiornet. Study of hydrocarbon migration models for basin simulators. Theses, Université d'Aix-Marseille, July 2011.

[49] S. S. Pegler, H. E. Huppert, and J. A. Neufeld. Fluid injection into a confined porous layer. J. Fluid Mech., 745:592-620, 2014.

[50] S. S. Pegler, H. E. Huppert, and J. A. Neufeld. Fluid migration between confined aquifers. J. Fluid Mech., 757:330-353, 2014.

[51] D. Pritchard. Gravity currents over fractured substrates in a porous medium. J. Fluid Mech., 584:415-431, 2007.

[52] D. Pritchard, A. W. Woods, and A. J. Hogg. On the slow draining of a gravity current moving through a layered permeable medium. J. Fluid Mech., 444:23-47, 2001.

[53] B. Schweizer. Homogenization of degenerate two-phase flow equations with oil trapping. SIAM J. Math. Anal., 39(6):1740-1763, 2008.

[54] Ø. Sylta. New techniques and their application in the analysis of secondary migration. In Basin Modelling: Advances and Applications, volume 3, pages 385-398. Elsevier, Amsterdam, 1993.

[55] D. Wilkinson. Percolation model of immiscible displacement in the presence of buoyancy forces. Phys. Rev. A, 30(1):520-531, 1984.

[56] D. Wilkinson and J. F. Willemsen. Invasion percolation: a new form of percolation theory. J. Phys. A: Math. Gen., 16:3365-3376, 1983.

[57] A. W. Woods and A. Farcas. Capillary entry pressure and the leakage of gravity currents through a sloping layered permeable rock. J. Fluid. Mech., 618: 361-379, 2009.

[58] B. Zhao, C. W. MacMinn, H. E. Huppert, and R. Juanes. Capillary pinning and blunting of immiscible gravity currents in porous media. Water Resour. Res., 50:7067-7081, 2014.

Clément Cancès (clement.cances@inria.fr), Inria, Univ. Lille, CNRS, UMR 8524 Laboratoire Paul Painlevé, F-59000 Lille.

David Maltese (david.maltese@univ-eiffel.fr), Laboratoire D'Analyse et De Mathématiques Appliquées (UMR 8050), Univ. Gustave Eiffel, UpeC, CNRS, F-77454, Marne-La-Vallée. 